\title{
THE STRUCTURE OF CERTAIN OPERATOR ALGEBRAS
}

\author{
BY \\ IRVING KAPLANSKY $\left({ }^{(1)}\right.$
}

1. Introduction. While the structure of commutative Banach algebras is today fairly well understood, the study of the noncommutative case is as yet in its infancy. It therefore appears to be in order to devote some attention to special classes of noncommutative Banach algebras. In this paper we have singled out a class which turns out to be comparatively accessible. These algebras (we call them $C C R$-algebras) are in the first place $C^{*}$-algebras (uniformly closed self-adjoint algebras of operators on Hilbert space); secondly we impose a hypothesis most briefly stated as follows: all the irreducible *-representations consist of completely continuous operators (the actual definition of $C C R$ in $\$ 5$ is given in a slightly different form, which is later shown to be equivalent).

Any commutative $C^{*}$-algebra is $C C R$, for the irreducible *-representations are then one-dimensional. The structure problem in the commutative case has been completely solved: one gets all the continuous complex functions vanishing at $\infty$ on a locally compact Hausdorff space. The main point of the present paper is that this kind of representation by continuous functions survives in a $C C R$-algebra, in a somewhat attenuated form. As a fundamental tool in setting up the representation, we employ the structure space introduced by Jacobson $[8]\left({ }^{2}\right)$ : the space $X$ of all primitive ideals, topologized by making the closure of $\left\{P_{i}\right\}$ the set of all primitive ideals containing $\cap P_{i}$. At every point $P$ of $X$ we thus have a primitive $C^{*}$-algebra $A / P$, and we may associate with any $a \in A$ the "function" whose value at $P$ is the homomorphic image $a(P)$ of $a \bmod P$. Naturally it is hopeless to speak of the continuity of $a(P)$, for we cannot compare elements in the unrelated algebras $A / P$. In special cases where the various algebras are not too badly unrelated, it was possible in [2] and [12] to get around this difficulty by utilizing a space other than the structure space. It does not seem to be possible to extend this device to the algebras studied in the present paper. But in any event there are some advantages in another attack which we now explain. Instead of $a(P)$ we consider $\|a(P)\|$; this is a real-valued function on $X$ whose continuity is perfectly meaningful. For many purposes this weakened continuity appears to suffice. It turns out (Theorem 4.1) that $\|a(P)\|$ is a continuous function for every $a \in A$ if and only if $X$ is a Hausdorff space.

Received by the editors December 10, 1949.

(1) Most of the work on this paper was done while the author was a fellow of the John Simon Guggenheim Memorial Foundation.

$\left.{ }^{2}\right)$ Numbers in brackets refer to the bibliography at the end of the paper. 
Now the structure space of a $C C R$-algebra $A$ is not necessarily Hausdorff, so this result is not directly applicable. We next introduce the idea of a composition series, confining ourselves exclusively to series which are wellordered ascending, and we are able to prove (Theorem 6.2) that $A$ has a composition series of closed ideals $I_{\rho}$ such that each $I_{\rho+1} / I_{\rho}$ has a Hausdorff structure space. This is our main structure theorem, and though it is by no means conclusive, it is strong enough to enable us to carry out (in \$7) a fairly complete study of ideals and subalgebras of a $C C R$-algebra. Actually the results can be pushed slightly beyond $C C R$-algebras to a class that we call GCR: a $G C R$-algebra is one having a composition series whose factor algebras are $C C R$.

The proof of Theorem 6.2 proceeds in several stages. First (Theorem 4.2) we treat the case where all $A / P$ are finite-dimensional and of the same order (or equivalently where all irreducible *-representations of $A$ are finite-dimensional and of the same degree). In Theorem 5.1 we show that the structure space of a $C C R$-algebra, while not necessarily Hausdorff, is at least of the second category. This information is used to make two further advances; first to the case where all $A / P$ are finite-dimensional, and then to the case of an arbitrary $C C R$-algebra.

Because of the intimate relation known to exist between locally compact groups and $C^{*}$-algebras, these results have applications to groups, which will be presented in a subsequent paper. The relevant groups (one might call them $C C R$-groups) have the property that for any irreducible unitary representation, the extension to the $L_{1}$-algebra consists of completely continuous operators. While it is not clear at this writing how far-reaching a class of groups this is, it is appropriate to note that the results of Gelfand and Neumark [5] show that the Lorentz group is a $C C R$-group.

In $\$ 2$ some preliminary results are derived concerning algebras of completely continuous operators. The results in $\$ 3$ are perhaps of independent interest; they are obtained by a systematic use of the technique of partitions. In Theorem 3.1 we prove, quite briefly, a general result concerning ideals in algebras of continuous functions; this theorem contains all previous results of this kind known to the author. Theorems 3.2-3.4 are devoted to noncommutative generalizations of the Weierstrass-Stone theorem. These considerations incidentally yield a new proof of the commutative WeierstrassStone theorem, the proof being of course based on partitions rather than the usual lattice-theoretic methods. The two commutative proofs are of about the same length; but partitions have the advantage of working in the noncommutative case, while lattices do not.

In $\$ 8$ we prove some results indicating that $C C R$-algebras behave in certain respects as if they had a unit element, and in $\$ 9$ the weakly closed case is considered. In the final section $(\$ 10)$ we turn from $C^{*}$-algebras to a nontopological analogue: algebraic algebras. Parallel, though not identical, argu- 
ments are applicable and yield a similar structure theory. An application is made to the problem raised by Kurosch as to whether every algebraic algebra is locally finite: it is shown (Theorem 10.4) that the answer is affirmative if it is affirmative for primitive algebras and nil algebras.

2. Dual $C^{*}$-algebras. We shall be speaking exclusively of Banach algebras with complex scalars, although nearly everything can be extended to the real case.

A $C^{*}$-algebra may be defined in either of the following ways: (1) a uniformly closed self-adjoint subalgebra of the algebra of all bounded operators on a Hilbert space, (2) a Banach algebra with a conjugate-linear involution *, satisfying the hypotheses that $\left\|x^{*} x\right\|=\|x\|^{2}$ and $x^{*} x$ has a quasi-inverse. The equivalence of these two definitions is due to Gelfand and Neumark [4], at least in the presence of a unit element; in [12, Theorem 7.1] the case where there is no unit was treated.

By a *-representation of a $C^{*}$-algebra we mean a ${ }^{*}$-preserving homomorphism into the algebra of bounded operators on a Hilbert space; a ${ }^{*}$-representation is necessarily continuous $[19$, p. 77$]$. A *-representation is trivial if every element is sent into the zero operator; it is irreducible if it is nontrivial and there are no proper closed invariant subspaces.

For the reader's convenience we restate two results [12, Theorems 6.4 and 7.2] for which we shall have frequent use; indeed the second of these will be constantly used without further reference. (We shall not be concerned in this paper with $B^{*}$-algebras in the sense of [12], and so we have restricted the following statements to $C^{*}$-algebras.)

Lemma 2.1. Let $\phi$ be an algebraic *-preserving isomorphism of a $C^{*}$-algebra $A$ into a dense subalgebra of a $C^{*}$-algebra $B$. Then $\phi$ maps $A$ onto $B$ and is an isometry.

Lemma 2.2. Let $I$ be a closed two-sided ideal in a $C^{*}$-algebra $A$. Then $A / I$ (in its natural norm and ${ }^{*}$-operation) is again a $C^{*}$-algebra.

We next recall a definition given in [12, p. 411]. If $A_{i}$ is a set of $C^{*}$-algebras, we define the $C^{*}$-sum of $\left\{A_{i}\right\}$ to be the set of all $\left\{a_{i}\right\}, a_{i} \in A_{i}$, with $\left\|a_{i}\right\|$ bounded; this is in a natural way again a $C^{*}$-algebra. More important is the $C^{*}(\infty)$-sum, which is the subset of all sequences vanishing at $\infty$, that is, all sequences $\left\{a_{i}\right\}$ such that for any $\epsilon>0$ all but a finite number of $\left\|a_{i}\right\|$ are less than $\epsilon$.

In [10] a dual ring was defined to be a topological ring in which for every closed right (left) ideal $I$ we have $R[L(I)]=I$ and $L[R(I)]=I$ respectively, where $L$ and $R$ denote the left and right annihilators. In [12, Theorem 8.3] the structure of a dual $C^{*}$-algebra was determined as follows:

Lemma 2.3. A dual $C^{*}$-algebra is the $C^{*}(\infty)$-sum of simple dual $C^{*}$-algebras (simple in the sense of having no proper closed two-sided ideals); a simple dual 
$C^{*}$-algebra is the algebra of all completely continuous operators on a suitable Hilbert space.

The converse of Lemma 2.3 is also valid. The first of the statements in Lemma 2.4 follows from [12, Theorem 8.4] and the second from [10, Theorems 6 and 7$]\left({ }^{3}\right)$.

Lemma 2.4. The algebra of all completely continuous operators on a Hilbert space is dual. The $C^{*}(\infty)$-sum of dual $C^{*}$-algebras is dual.

The definition of a dual ring by the properties of annihilators will not be at all relevant in what is to follow; however the designation "dual" has the merit of brevity, and we shall stick to it. The following two alternative characterizations of dual $C^{*}$-algebras are more useful.

THEOREM 2.1. The following statements are equivalent for a $C^{*}$-algebra $A$ : (1) $A$ is dual, (2) $A$ has a faithful *-representation by completely continuous operators, (3) the socle of $A$ is dense (the socle is the union of the minimal right ideals).

Proof. That (1) implies (2) follows from Lemma 2.3: by taking the (Hilbert space) direct sum of the Hilbert spaces on which the simple components of $A$ are represented, we get in a natural way a faithful representation of $A$ by completely continuous operators.

$(2) \rightarrow(3)$. Suppose that $A$ is a $C^{*}$-algebra of completely continuous operators and let $S$ be the closure of its socle. The spectral theorem for completely continuous operators asserts that a self-adjoint element $x$ in $A$ is a limit, in the uniform topology, of linear combinations of finite-dimensional projections. The latter are in $A$, being uniform limits of polynomials in $x$, and moreover they are in $S$ (the right ideal generated by a finite-dimensional projection is the union of a finite number of minimal right ideals). Hence $x \in S$ and $S=A$.

$(3) \rightarrow(1)$. Suppose that the socle of $A$ is dense. Let $\left\{I_{n}\right\}$ denote the distinct minimal two-sided ideals of $A, J_{n}$ the closure of $I_{n}, K_{n}$ the closure of the union of the remaining $I^{\prime}$ s, $B$ the $C^{*}(\infty)$-sum of the $J^{\prime}$ s. By [12, Theorem 7.3], $J_{n}$ is the algebra of all completely continuous operators on a Hilbert space. By Lemma $2.4, B$ is dual. We shall prove that $A$ is dual by establishing its isomorphism with $B$. We know that $I_{m} I_{n}=0(m \neq n)$ and consequently $J_{n} K_{n}=0$. Then $J_{n} \cap K_{n}=0$ (a $C^{*}$-algebra has no nil ideals). Next $J_{n}+K_{n}$ is dense in $A$ since it contains at least the socle. By [12, Lemma 8.1] $J_{n}+K_{n}$ is all of $A$, and $A$ is the direct sum of $J_{n}$ and $K_{n}$. Let $x_{n}$ be the $J_{n^{-}}$ component of $x$ in this direct sum. The mapping $x \rightarrow\left\{x_{n}\right\}$ is an isomorphism

(3) To apply [10, Theorem 7] we have to know that $x$ is in the closure of $x A$. This is true for any $C^{*}$-algebra $A$; in fact, the argument of [12, Theorem 7.2] shows that $x$ is in the closed right ideal generated by $x x^{*}$. 
that sends at least the socle of $A$ into $B$, for, if $x$ is in the socle, all but a finite number of $x_{n}$ 's vanish. It follows that all of $A$ is mapped into $B$ and by Lemma 2.1 the mapping is onto and an isometry.

The criterion (2) of Theorem 2.1 gives us an immediate corollary.

Corollary. A closed self-adjoint subalgebra of a dual $C^{*}$-algebra is dual.

Before proceeding to the next theorem, we prove a lemma in somewhat greater generality than needed.

LemMA 2.5. Let $A$ be an irreducible algebra of operators on a Banach space $E$. Then $A$ has no ideal divisors of 0 , that is, if $I$ and $J$ are two-sided ideals in $A$ with $I J=0$, then $I$ or $J=0$.

Proof. Let $F$ be the closed subspace spanned by the ranges of the operators in $I$. Then $F$ is a closed invariant subspace, and it is annihilated by $J$. If $F=0$, then $I=0$. If $F=E$, then $J=0$.

The following theorem is now proved in preparation for a later study of the Weierstrass-Stone theorem. We remark that the theorem is actually true with $A$ any dual $C^{*}$-algebra; in fact a good deal more will be proved in Theorem 7.2. But for the present this special case will suffice.

TheOREM 2.2. Let $A$ be a dual $C^{*}$-algebra which is either simple or the direct sum of two simple algebras. Let $B$ be a closed self-adjoint subalgebra with the following property: for any distinct regular maximal right ideals $M$ and $N$ in $A, B$ contains an element in $M$ but not in $N$. Then $B=A$.

Proof. First we consider the case where $A$ is simple. We can then suppose $A$ concretely given as the algebra of all completely continuous operators on a Hilbert space $H$. We assert that the induced representation of $B$ is irreducible. For suppose on the contrary that $H=F \oplus G$ with $F, G$ invariant under $B$. Let $\alpha, \beta$ be nonzero vectors in $F, G$ and let $M, N$ be the (regular maximal) right ideals in $A$ which annihilate $\alpha+\beta$ and $\alpha-\beta$ respectively. Then any operator in $B \cap M$ or $B \cap N$ must annihilate both $\alpha$ and $\beta$, and so $B$ cannot distinguish $M$ and $N$. Hence the representation of $B$ on $H$ is irreducible. By the corollary to Theorem 2.1, $B$ is dual. By Lemma 2.5, $B$ has no ideal divisors of 0 . It follows that $B$ cannot be a nontrivial direct sum, and so by Lemma 2.3, $B$ is simple. Now Neumark [15] has shown that, up to unitary equivalence, a simple dual $C^{*}$-algebra has precisely one irreducible representation, and it consists of course of all completely continuous operators on the Hilbert space in question. Hence $B=A$.

Suppose now that $A$ is the direct sum of two ideals $I$ and $J$, each of which is a simple dual $C^{*}$-algebra. Let us write $I_{1}=B \cap I, J_{1}=B \cap J$. The algebra $B / I_{1}$ can, in a natural way, be regarded as a subalgebra of $A / I$, the mapping being implemented by sending the coset $b+I_{1}$ into the coset $b+I$. Let $M_{0}$, $N_{0}$ be regular maximal right ideals in $A / I$, and let $M, N$ be their inverse 
images in $A$; they will again be regular maximal. By hypothesis, $B$ contains an element $x$ in $M$ but not in $N$. The homomorphic image of $x \bmod I_{1}$ is an element of $B / I_{1}$ lying in $M_{0}$ but not in $N_{0}$. It follows that $B / I_{1}$ satisfies the hypothesis of our theorem relative to the dual simple algebra $A / I$. By the preceding paragraph, $B / I_{1}$ is all of $A / I_{1}$. This can be translated to say $B+I=A$.

We look again at the ideals $I_{1}, J_{1}$ and observe $I_{1} \cap J_{1}=0$. Next, $I_{1}+J_{1}$ is closed [12, Lemma 8.1]. But since $B / I_{1}$ is simple, there are no closed ideals between $I_{1}$ and $B$. Hence either (1) $I_{1}+J_{1}=I_{1}=J_{1}$, or (2) $I_{1}+J_{1}=B$. (1) In this case $I_{1}=J_{1}=0$, and so $B$ is simple. We return to the algebras $I, J$ and note that they have representations as all completely continuous operators on Hilbert spaces, say $H$ and $K$. In a natural way this induces a representation of $A$, and hence $B$, on $H \oplus K$. In this representation of $B$, it will still be true that $H$ and $K$ are irreducible; the proof is virtually the same as the corresponding one in the second last paragraph. By the theorem of Neumark cited above, these two irreducible representation of $B$ on $H$ and $K$ are unitarily equivalent. Let $\gamma, \delta$ be nonzero vectors of $H$ and $K$ which correspond under this unitary equivalence. Let $M^{\prime}, N^{\prime}$ be the (regular maximal) right ideals in $A$ which annihilate $\gamma$ and $\delta$ respectively. Then $B$ is unable to distinguish $M^{\prime}$ and $N^{\prime}$, and we have a contradiction.

We are thus led to conclude that case (2) holds: $I_{1}+J_{1}=B$. Combining this with $B+I=A$, we have $I_{1}+J_{1}+I=A, J_{1}+I=A$. This is possible only if $J_{1}=J, B \supset J$. Similarly $B \supset I$ and $B=A$.

In concluding this section we remark that the study of dual $C^{*}$-algebras can be pursued further, and that they appear to be nearly as well behaved as finite-dimensional algebras. In particular, the classical theorems about pairs of commutators and extensions of isomorphisms have satisfactory analogues.

3. Partitions.' Much of our later work will rest on results obtainable by a suitable application of partitions. We state the requisite topological lemma in the form given by Bourbaki [3, p. 65].

Leмma 3.1. Let $X$ be a normal Hausdorff space and $U_{1}, \cdots, U_{n}$ a covering of $X$ by open sets. Then: (a) there exists a covering by open sets $V_{1}, \cdots, V_{n}$ such that the closure of $V_{i}$ is in $U_{i}$, (b) we may write $1=f_{1}+\cdots+f_{n}$ where $f_{i}$ is a real non-negative continuous function on $X$ vanishing outside $U_{i}$.

We are now going to apply partitions to the study of algebras of functions. For the purpose of later applications, it is essential to take up the case of functions whose value $f(x)$ at $x$ lies in an algebra $A_{x}$ depending on $x$. In doing this, we shall follow Godement [6], with some slight modifications.

Let then $X$ be a compact Hausdorff space and for each $x \in X$ let there be given a Banach algebra $A_{x}$. The case of functions "vanishing at $\infty$ " can be covered by allowing one or more $A_{x}$ 's to be 0 (there is actually no loss of generality in insisting that there be at most one $\left.A_{x}=0\right)$. Write $C(X)$ for the 
algebra of all real continuous functions on $X$. We consider an algebra $A$ of functions $f$ on $X$ with $f(x) \in A_{x}$, and for the purposes of Theorem 3.1, we impose four conditions:

(a) $\|f(x)\|$ is continuous on $X$,

(b) $A$ is complete under the norm $\|f\|=\sup \|f(x)\|$,

(c) at each point $x, A$ fills out $A_{x}$,

(d) $A$ is closed under multiplication by $C(X)$, and moreover the result of multiplying $f \in A$ by an element of $C(X)$ lies in the closed right ideal generated by $f$.

We pause to assess the significance of the assumption (d). The simplest way of fulfilling it is of course to assume outright that $A$ contains $C(X)$, or at any rate contains all continuous real functions that vanish at the points $x$ where $A_{x}=0$. As a preliminary application of partitions, we shall now show that under suitable circumstances we can weaken (d) to the mere assumption that $A$ is closed under multiplication by $C(X)$.

Lemma 3.2. Let $X$ be a compact Hausdorff space at each point of which a Banach algebra $A_{x}$ is given. Suppose that each $A_{x}$ satisfies the condition that every $z \in A_{x}$ lies in the closure of $z A_{x}\left({ }^{4}\right)$. Let $A$ be an algebra of functions $f$ on $X$ with $f(x) \in A_{x}$, satisfying postulates (a), (b), and (c) above, and further satisfying the portion of (d) that asserts closure of $A$ under multiplication by $C(X)$. Then $A$ satisfies all of $(\mathrm{d})$.

Proof. Let $f \in A, \lambda \in C(X)$, and a positive $\epsilon$ be given. For each $x \in X$ we can find an element $g \in A$ whose value $g(x)$ at $A_{x}$ satisfies $\|f(x) g(x)-f(x)\|<\epsilon$. In a suitable neighborhood $U$ of $x$ this continues to be true. Select a finite covering of $X$, say by $U_{1}, \cdots, U_{n}$, and corresponding elements $g_{1}, \cdots, g_{n}$. By Lemma 3.1, select a partition of unity relative to the $U$ 's, that is, write $1=\theta_{1}+\cdots+\theta_{n}$ with $\theta_{i}$ continuous, non-negative and vanishing outside $U_{i}$. By hypothesis, $h=\lambda \sum \theta_{i} g_{i}$ is a well defined element of $A$. We assert

$$
\|f h-\lambda f\|<\epsilon \text {. }
$$

The argument for this is standard, and we shall give it only on this first occasion. To prove (1), we must verify it at the general point $y$ of $X$. Suppose for definiteness that $y$ lies in $U_{1}, \cdots, U_{r}$, but not in $U_{r+1}, \cdots, U_{n}$. Then

$$
\left\|f(y) g_{i}(y)-f(y)\right\|<\epsilon
$$

holds for $i=1, \cdots, r$. Moreover at $y, \theta_{r+1}=\cdots=\theta_{n}=0$, and $\theta_{1}, \cdots, \theta_{r}$ are non-negative and add up to 1 . On taking the appropriate convex combination of the elements (2), we find that the result is still less than $\epsilon$ :

$$
\left\|\sum \theta_{i}(y)\left[f(y) g_{i}(y)-f(y)\right]\right\|<\epsilon .
$$

(4) This hypothesis is of course satisfied if $A_{x}$ has a unit element; it is also satisfied if $A_{x}$ is a $C^{*}$-algebra (footnote 3 ). 
A further multiplication of (3) by $\lambda(y)$ yields (1) evaluated at $y$. Hence (1) is proved, and it shows that $\lambda f$ can be approximated arbitrarily closely by right multiples of $f$, as desired.

We now prove a general result concerning the form of closed ideals in algebras of functions.

TheOREM 3.1. Let $X$ be a compact Hausdorff space at each point of which a Banach algebra $A_{x}$ is given. Let $A$ be an algebra of functions $f$ on $X$ with $f(x)$ $\in A_{x}$, satisfying postulates (a) to (d) above. Then any closed right ideal I in $A$ has the following form: for each $x$ a closed right ideal $I_{x}$ in $A_{x}$ is given, and $I$ consists of all $f \in A$ with $f(x) \in I_{x}$ for all $x$.

Proof. Let $H_{x}$ be the set of all values taken at $x$ by members of $I ; H_{x}$ is in any event a right ideal. A priori $H_{x}$ might not be closed, and we let $I_{x}$ be its closure. Let $h \in A$ be an element with $h(x) \in I_{x}$; we must prove $h \in I$. It will suffice to approximate $h$ in $I$ within $\epsilon$. For any $x \in X$ we can find $g \in I$ such that $g(x)$ is within $\epsilon$ of $h(x)$. This continues to be true in a suitable neighborhood of $x$. A finite number of these neighborhoods cover $X$, say $U_{1}, \cdots, U_{n}$. Let $\theta_{1}, \cdots, \theta_{n}$ be a corresponding partition of unity (Lemma 3.1). Then $\sum \theta_{i} g_{i} \in I$ (we are using hypothesis (d) and the fact that $I$ is closed). The observation $\left\|\sum \theta_{i} g_{i}-h\right\|<\epsilon$ concludes the proof.

Many corollaries can be deduced from Theorem 3.1. Without attempting to be exhaustive, we shall mention three; for each of them the hypothesis of Theorem 3.1 is assumed to be in force.

Corollary 1. Any closed maximal (right) ideal in A consists of all functions confined at a certain point $x$ to a closed maximal (right) ideal in $A_{x}$.

Corollary 2. If each $A_{x}$ is simple (that is, has no proper closed two-sided ideals), then a closed two-sided ideal in $A$ consists of all functions vanishing on a closed subset of $X$.

COROLlary 3. If in each $A_{x}$ it is true that a closed right ideal is the intersection of the regular maximal right ideals containing it, then the same is true in $A$.

A fourth corollary is a fairly immediate consequence of Lemma 3.2 and Theorem 3.1.

CoROllary 4. Let $B$ be a Banach algebra with the property that for any $z \in B$, $z$ is in the closure of $z B$. Let $X$ be a locally compact Hausdorff space, and $A$ the $B$ anach algebra of all continuous functions vanishing at $\infty$ from $X$ to $B$. Then any closed right ideal in $A$ has the structure described in Theorem 3.1.

Remark. One can carry out a similar discussion of the case where each $A_{x}$ is merely a suitable locally convex topological algebra, for example, the algebra of all bounded operators on a Banach space in its strong or weak topology. Thus generalized, Theorem 3.1 subsumes a result of Hing Tong 
[20, Theorem 7]. It is possible further to consider the case where $A_{x}$ is a locally convex topological linear space; Theorem 3.1 then describes the closed $C(X)$ submodules of $A$.

We are now going to specialize the discussion of algebras of functions, by requiring that each $A_{x}$ be a $C^{*}$-algebra. Before doing so, several remarks on $C^{*}$-algebras are in order.

(1) We use the definition of the spectrum given in [12]: if $x$ is an element of a Banach algebra $A$, the nonzero spectrum of $x$ consists of all scalars $\lambda$ such that $-\lambda^{-1} x$ does not have a quasi-inverse, and we insert 0 in the spectrum unless $A$ has a unit element and $x$ has an inverse with respect to it. The insertion of 0 in the spectrum is handled differently by other authors, but the ambiguity is not of great importance. However we take the definition, there are special difficulties at 0 , as is illustrated by Lemma 3.3 below.

(2) Let $A$ be a $C^{*}$-algebra and $x$ a self-adjoint element of $A$. The closed subalgebra $B$ generated by $x$ is known to consist of all continuous complex functions vanishing at $\infty$ on a locally compact Hausdorff space $X$ (in fact $X$ is homeomorphic to the nonzero spectrum of $x$ ). Let $p$ be any continuous real function with $p(0)=0$. Then $p(x)$ is a well defined element of $B$ (and is again self-adjoint). This technique of applying a scalar continuous function to a self-adjoint element will be used repeatedly.

Suppose further that $I$ is a closed two-sided ideal in $A$ and let $x^{\prime}$ be the homomorphic image of $x \bmod I$. Then $p\left(x^{\prime}\right)$ may be formed, and we assert that it is the homomorphic image of $p(x)$. This is clear if $p$ is a polynomial (with no constant term of course). The general $p$ is a uniform limit of such polynomials, and the result follows from the continuity of the map from $A$ to $A / I$.

(3) As an application of the preceding remark, we prove a result which will be used later. Let $A$ be a $C^{*}$-algebra, $I_{n}$ a set of closed two-sided ideals with intersection 0 , and $x$ a self-adjoint element in $A$ such that each image $x_{n} \bmod I_{n}$ satisfies $x_{n} \geqq 0$; then $x \geqq 0$. To see this, define $p$ by $p(t)=0$ for $t \geqq 0, p(t)=-t$ for $t \leqq 0$. Then $p\left(x_{n}\right)=0$, hence $p(x)=0$, hence $x \geqq 0$. (By a slight extension of this remark, one can prove that the spectrum of $x$ is the closure of the union of the spectra of $x_{n}$.)

(4) Suppose finally that we have a self-adjoint $C^{*}$-algebra $A$ of functions from $X$ to $C^{*}$-algebras $A_{x}$, as described below. Then (by considering the ideals of functions vanishing at a given coordinate) it follows from the above that: (a) in applying a continuous real function $p$ to an element in $A$, the result is obtained by applying $p$ coordinate-wise, (b) if a self-adjoint element of $A$ is not less than 0 at each coordinate, then it is not less than 0 in $A$.

Let now $X$ be any topological space at each point of which a $C^{*}$-algebra $A_{x}$ is given. If we wish to combine these to get a $C^{*}$-algebra $A$ of functions, it is in the first place necessary to assume that $A$ is self-adjoint, that is, contains $f^{*}$ along with $f, f^{*}$ being defined by taking the * pointwise. Besides this, 
we are going to analyze more closely the assumptions (a)-(d) above. We begin by observing that the condition,

$\left(\mathrm{b}^{\prime}\right)\|f(x)\|$ is bounded, and $A$ is complete under the norm $\|f\|=\sup \|f(x)\|$, suffices to ensure that a self-adjoint algebra $A$ is a $C^{*}$-algebra. (In other words, we are taking $A$ to be a closed self-adjoint subalgebra of the $C^{*}$-sum of the algebras $A_{x}$.) We next single out a piece of continuity:

$\left(\mathrm{a}^{\prime}\right)\|f(x)\|$ is continuous at 0 , that is, if $f(x)=0$, then for any $\epsilon>0$, there exists a neighborhood $U$ of $x$ such that $\|f(x)\|<\epsilon$ for $x$ in $U$.

From this assumption we proceed to deduce (for later use) a sort of continuity of the spectrum.

Lemma 3.3. Let $X$ be a topological space at each point of which a $C^{*}$-algebra $A_{x}$ is given, and let $A$ be a self-adjoint algebra of functions from $X$ to $\left\{A_{x}\right\}$, with $f(x) \in A_{x}$, satisfying $\left(\mathrm{a}^{\prime}\right)$ and $\left(\mathrm{b}^{\prime}\right)$ above. Then for any self-adjoint element $f \in A$ the spectrum of $f(x)$ is a continuous function of $x$ in the following sense: for any $x \in X$ and $\epsilon>0$ there is a neighborhood $U$ of $x$ such that for all $y \in U$, the spectrum of $f(y)$ is contained in an $\epsilon$-neighborhood of the set consisting of 0 and the spectrum of $f(x)$.

Proof. Write $V$ for the set consisting of 0 and the spectrum of $f(x)$, and $W$ for an $\epsilon$-neighborhood of it. Let $p$ be a continuous real-valued function which vanishes on $V$ and is equal to 1 on the complement of $W$. Then $g=p(f)$ vanishes at $x$, and so by the continuity of the norm, $\|g(y)\|<1$ for $y$ in a suitable neighborhood $U$ of $x$. For $y$ in $U$ the spectrum of $g(y)$ must lie in $W$.

We now turn our attention to assumption (d) above. In view of Lemma 3.2, and the fact that the hypothesis on $A_{x}$ in that lemma is satisfied if each $A_{x}$ is a $C^{*}$-algebra, we are concerned only with the part of assumption (d) asserting closure under multiplication by $C(X)$. In the next two theorems we show that it is possible to derive this closure under $C(X)$ from weaker assumptions about what the algebra contains at pairs of points. When specialized to the commutative case, a statement of this kind coincides with the Weierstrass-Stone theorem, and so we may regard Theorems 3.2-3.4 as noncommutative generalizations of the Weierstrass-Stone theorem.

It turns out that the simplest case to handle is that where each $A_{x}$ has a unit element. We denote them all ambiguously by 1 , and it then becomes meaningful to formulate another piece of continuity:

$\left(\mathrm{a}^{\prime \prime}\right)\|f(x)\|$ is continuous at 1 , that is, if $f(x)=1$ then for any $\epsilon>0$ there exists a neighborhood $U$ of $x$ such that $\|f(y)-1\|<\epsilon$ for $y$ in $U$. We use this as part of the hypothesis in the following theorem.

THEOREM 3.2. Let $X$ be a compact Hausdorff space at each point of which a $C^{*}$-algebra $A_{x}$ is given. We suppose that each $A_{x}$ has a unit element, but we admit the possibility of an exceptional point $u$ where $A_{u}=0$. Let $B$ be a self-adjoint algebra of functions $f$ on $X$ with $f(x) \in A_{x}$; we suppose that $\left(\mathrm{a}^{\prime}\right),\left(\mathrm{a}^{\prime \prime}\right)$, and $\left(\mathrm{b}^{\prime}\right)$ above are satisfied, that is, $\|f(x)\|$ is bounded and continuous at 0 and 1 , and $B$ 
is complete under the sup norm. Suppose further that for any distinct points $y$ and $z(z \neq u), B$ contains a function $f$ with $f(y)=0, f(z)=1$. Write $C^{\prime}(X)$ for the algebra of real continuous functions on $X$, vanishing at the exceptional point $u$ (if there is one). Then, $B$ contains $C^{\prime}(X)$.

Proof. The idea of the proof is to approximate an element of $C^{\prime}(X)$ by the use of a partition. However it would be futile to select a partition in the ordinary way, since its members would not be known to be in $B$; a priori, $B$ is not known to contain any elements of $C^{\prime}(X)$ at all. Instead we build a partition out of whatever is available in $B-\mathrm{a}$ "noncommutative" partition, so to speak.

It is convenient to suppose that an exceptional point $u$ is really presentwe can adjoin an isolated point if necessary. Let $C$ be the $C^{*}$-algebra obtained by adjunction of a unit element to $B$; it consists of all functions obtained by adding constant scalar functions to $B$. If we prove that $C$ contains all real continuous functions on $X$, we will know that $B$ contains those vanishing at $u$. We note that continuity of $\|f(x)\|$ at 0 and 1 survives in the enlarged algebra $C$ (as a matter of fact we shall need continuity only at 0 in $C$, but to get this requires continuity at both 0 and 1 in $B$ ).

In this paragraph we shall prove that $C$ contains a function equal to 0 and 1 respectively on prescribed disjoint compact subsets of $X$. We know that for any distinct points $y$ and $z, C$ contains a self-adjoint element $k$ with $k(y)=0, k(z)=1$. Let $p$ be a real-valued continuous function which vanishes in a neighborhood of 0 and satisfies $p(1)=1$. Then $k_{1}=p(k)$ vanishes in a neighborhood of $y$ and still satisfies $k_{1}(z)=1$. Let $K$ be a compact set disjoint from $z$. For each of its points we find an element in $C$ vanishing in a neighborhood of the point and equal to 1 at $z$. A finite number of these neighborhoods cover $K$. Multiplication of the corresponding functions gives us one which vanishes on all of $K$ and is equal to 1 at $z$. Finally, let $K$ and $L$ be disjoint compact sets. For any point $z$ in $L$, take the above constructed $k_{2}$ which vanishes on $K$ and satisfies $k_{2}(z)=1$; we may assume $k_{2}$ self-adjoint. With the same real-valued function $p$ as above, form $k_{3}=p\left(1-k_{2}\right) ; k_{3}=1$ on $K$ and vanishes in a neighborhood of $z$. We do this at every point of $L$, select a finite covering of $L$ by the resulting neighborhoods, and multiply the corresponding functions $k_{3}$. The result is the desired function in $C$ equal to 1 and 0 on $K$ and $L$.

Now let $h$ be a continuous real function on $X$. We propose to approximate it in $C$ within $\epsilon$. Decompose $X$ into open sets $U_{1}, \cdots, U_{n}$ on which the oscillation of $h$ is less than $\epsilon$, and let $r_{i}$ be one of the values assumed by $h$ in $U_{i}$. Let $V_{1}, \cdots, V_{n}$ be a covering of $X$ by open sets with the closure of $V_{i}$ in $U_{i}$-we are citing part (a) of Lemma 3.1. By the preceding paragraph, $C$ contains a function $d_{i}$ which equals 1 on $V_{i}$ and 0 on the complement of $U_{i}$. Write $e_{i}=d_{i}^{*} d_{i}, e=\sum e_{i}$. Then $e \geqq 1$ everywhere, and it follows (see the remarks above) that $e \geqq 1$ also holds in $C$. From this we deduce that $e$ has an 
inverse in $C$. Moreover that inverse is again positive definite, and has a unique positive square root $g$, so that $g^{2}=e^{-1}$. We write $f_{i}=g e_{i} g$, and observe that $f_{i}$ is self-adjoint, $f_{i} \geqq 0$, and $\sum f_{i}=1$. Then $\sum r_{i} f_{i}$ is in $C$, and we assert that it approximates $h$ within $\epsilon$. Since the convex combination here involves ring elements, this claim needs further substantiation beyond the standard argument. So let $x$ be any point in $X$, lying say in $U_{1}, \cdots, U_{m}$, but not in $U_{m+1}, \cdots, U_{n}$. We have that for $i \leqq m, r_{i}-h(x)$ is a real number $s_{i}$ with $\left|s_{i}\right|<\epsilon$. Now choose a fixed operator ${ }^{*}$-representation of the $C^{*}$-algebra $A_{x}$, representing $f_{i}(x)$ say by $T_{i}$; we have $T_{i} \geqq 0$ and $T_{1}+\cdots+T_{m}=$ the identity operator. Our task is to prove that $\left\|\sum r_{i} f_{i}-h\right\|<\epsilon$ holds at the point $x$, and this is equivalent to $\left\|\sum s_{i} T_{i}\right\|<\epsilon$. For this purpose it suffices to show that

$$
\left|\left(\alpha \sum s_{i} T_{i}, \alpha\right)\right|=\left|\sum s_{i}\left(\alpha T_{i}, \alpha\right)\right|<\epsilon
$$

holds for any vector $\alpha$ of length 1 . But for $i=1, \cdots, m$ the numbers $\left(\alpha T_{i}, \alpha\right)$ are real non-negative and have sum $=1 ;(4)$ then follows from $\left|s_{i}\right|<\epsilon$. This concludes the proof of Theorem 3.2.

Remarks. 1. The assumption $\left(a^{\prime \prime}\right)$ of continuity at 1 cannot be dropped in Theorem 3.2. An example showing this is the following: take $A$ to be the algebra of all sequences of 2 by 2 matrices approaching a matrix of the form

$$
\left(\begin{array}{ll}
a & 0 \\
0 & 0
\end{array}\right) \text {. }
$$

$A$ is in a natural way a $C^{*}$-algebra of functions on a space $X$ consisting of a sequence and a limit point. The norm is continuous on $X$ (not just at 0 , but unrestrictedly). However the norm is not continuous at 1 in the sense of $\left(\mathrm{a}^{\prime \prime}\right)$, and though $A$ satisfies all the other hypotheses of Theorem 3.2, it fails to contain $C(X)$. On the other hand, $A$ does admit multiplication by $C(X)$, and this checks with Theorem 3.3 below.

This same example, incidentally, illustrates that the precautions taken at 0 in Lemma 3.3 were indispensable.

2. If it is the case that not only does each $A_{x}$ have a unit, but $A$ itself has a unit, then $\left(a^{\prime \prime}\right)$ follows from $\left(a^{\prime}\right)$.

3. The assumption in Theorem 3.2 of a unit element in each $A_{x}$ is alarmingly restrictive for some applications. Whether a theorem like 3.3 or 3.4 can be proved without any restriction on the $A$ 's is not known to the author. However a satisfactory result is available in the case where each $A_{x}$ is dual. In connection with Theorem 3.3, we note: (a) it seems to be necessary to revert to hypothesis (a) above, full-blown continuity of the norm; (b) since there are no unit elements in sight, closure under multiplication by $C(X)$ is the strongest conclusion we can hope for.

THEOREM 3.3. Let $X$ be a compact Hausdorff space at each point of which 
a dual $C^{*}$-algebra $A_{x}$ is given (some of the $A_{x}$ 's may be 0 ). Let $B$ be a self-adjoint algebra of functions $f$ on $X$ with $f(x) \in A_{x}$, satisfying postulates (a) and (b) above, that is, $\|f(x)\|$ is continuous and $B$ is complete under the sup norm. Suppose further that for any distinct points $x, y \in X, B$ contains functions taking arbitrary pairs of values in $A_{x}, A_{y}$ at $x, y$. Then $B$ is closed under multiplication by $C(X)$, the algebra of all real continuous functions on $X$.

Proof. Let an element $g$ of $B, \lambda(x) \in C(X)$, and a positive $\epsilon$ be given. Write $h=g_{*} g$. We pick a point $y$ in $X$ and examine the spectrum of $h(y)$. We shall treat explicitly the case where this spectrum is infinite; with some slight modifications the argument applies to the case where it is finite. Then the spectrum of $h(y)$ consists of 0 and a descending sequence $\alpha_{1}, \alpha_{2}, \cdots$ of positive real numbers approaching 0 -this follows from Theorem 2.1 and known facts about completely continuous operators. Suppose $\alpha_{r}<\epsilon$, and choose $\beta$ and $\gamma$ with $\alpha_{r}>\beta>\gamma>\alpha_{r+1}$. Define $p(t)$, a real-valued function of the real variable $t$ as follows: $p(t)=0$ for $t \leqq \gamma, p(t)=1$ for $t \geqq \beta$, and $p$ is linear between $\gamma$ and $\beta$. Write $e=p(h)$. At the point $y$ we have $\|e h-h\|<\epsilon$, and (by the hypothesis of continuity of the norm) this persists in a suitable neighborhood $U_{1}$ of $y$. Further, Lemma 3.3 shows that for $z$ in a neighborhood $U_{2}$ of $y$, the spectrum of $h(z)$ lies outside $(\gamma, \beta)$; it follows that $e(z)$ is an idempotent for $z$ in $U_{2}$. Let $U=U_{1} \cap U_{2}$, and let $V$ be an open set containing $y$, with closure $V^{\prime}$ in $U$.

In this paragraph we shall show that, within $V^{\prime}, e$ can be multiplied by an arbitrary continuous real function. For this purpose we introduce $I$, the (closed two-sided) ideal in $B$ consisting of all functions vanishing on $V^{\prime}$. Let $e_{1}$ denote the image of $e \bmod I$. We consider the algebra $C=e_{1}(B / I) e_{1}$. In a natural way, $C$ admits a functional representation on the space $V^{\prime}$, with the functional values at $x \in V^{\prime}$ lying in $e(x) A_{x} e(x)$, and it is still true that for any distinct points $x, w$ in $V^{\prime}, C$ contains a function taking any prescribed pair of values in $e(x) A_{x} e(x)$ and $e(w) A_{w} e(w)$. Moreover the continuity and completeness conditions-labelled (a) and (b) above-are still satisfied. All the more so are the hypotheses $\left(\mathrm{a}^{\prime}\right)$ and $\left(\mathrm{b}^{\prime}\right)$ of Theorem 3.2 fulfilled. Finally, $C$ has $e_{1}$ as unit element, and from this (remark 2 above) we deduce that $C$ satisfies the hypothesis $\left(a^{\prime \prime}\right)$ of continuity of the norm at 1 . All the hypotheses of Theorem 3.2 have now been verified and we are entitled to conclude that $C$ contains all continuous real functions on $V^{\prime}$, or more precisely, all multiples of $e_{1}$ by continuous real functions on $V^{\prime}$. By taking the inverse image in $B$ of the elements just constructed, we achieve the desired elements which are, within $V^{\prime}$, multiples of $e$ by arbitrary real continuous functions on $V^{\prime}$.

While the functions just constructed behave well on $V^{\prime}$, we have as yet no control over them outside $V^{\prime}$. We shall now adjust this situation, by an argument like that used in Theorem 3.2. Let $W$ be an open set with closure $W^{\prime}$ in $V, y \in W$. We are going to construct an element $f$ in $B$ such that $f=e$ on $W^{\prime}, f=0$ on the complement $Z$ of $V$. Reference to the construction of $e$ as $p(h)$ shows that for any $z$ in $Z$, the spectrum of $e(z)$ is finite. Hence there 
exists a self-adjoint element $d$ in $B$ such that $d(z)$ is an idempotent and a unit element for $e(z)$, and we can further suppose that $d$ vanishes at a selected point $w$ of $W^{\prime}$. Apply to $d$ a (real-valued continuous) function which vanishes in a neighborhood of 0 and takes the value 1 at 1 ; this will not change $d$ at $z$ and will make it vanish in a neighborhood of $w$. A covering of $W^{\prime}$ reduced to a finite covering, plus a multiplication of the resulting functions, gives us an element vanishing on $W^{\prime}$ and satisfying $d_{1}(z)=d(z)$. Now consider $e-d_{1} e$; this coincides with $e$ on $W^{\prime}$ and vanishes at $z$. It should be clear how another application of the same device gives us the desired element $f$-vanishing on $Z$ and equal to $e$ on $W^{\prime}$.

We next put our two results together. Let $\theta$ be a real continuous function on $X$ which vanishes outside $W$. By our first result we know that $B$ contains an element which on $V^{\prime}$ coincides with $\theta e$. Multiply this element by $f$ and we have one which coincides everywhere with $\theta e$. In short, $e$ admits multiplication by real continuous functions vanishing on the complement of $W$.

We are now ready for the final construction. The neighborhood $W$ and its associated element $e$ have been constructed relative to the point $y$. Do this at every point of $X$ and extract a finite covering, say by sets $W_{1}, \cdots, W_{r}$ with associated elements $e_{1}, \cdots, e_{r}$. Split 1 by a partition: $1=\theta_{1}+\cdots+\theta_{r}$, where $\theta_{i}$ is real continuous, non-negative on $X$ and vanishes outside $W_{i}$. Then, by the preceding paragraph, $\lambda \theta_{i} e_{i}$ is a well defined element of $B$. Write $k=\lambda g\left(\theta_{1} e_{1}+\cdots+\theta_{r} e_{r}\right)$. Then

$$
(k-\lambda g)^{*}(k-\lambda g)=\lambda^{2} \sum_{i, j} \theta_{i} \theta_{j}\left(e_{i}-1\right) h\left(e_{j}-1\right) .
$$

In view of the fact that $\left\|e_{i} h-h\right\|<\epsilon$ in $W_{i}$ this gives

$$
\|k-\lambda g\|^{2} \leqq[\sup |\lambda|]^{2} \epsilon \text {, }
$$

and shows that we can approximate $\lambda g$ arbitrarily closely in $B$. This concludes the proof of Theorem 3.3.

We note (for later use) a corollary of Theorem 3.3. We first cite [12, Theorem 8.4] and deduce from it that every closed right ideal in a simple dual $C^{*}$-algebra is an intersection of regular maximal right ideals. Next, this result extends to any dual $C^{*}$-algebra, that is, to a $C^{*}(\infty)$-sum of simple dual $C^{*}$-algebras; this can be seen directly, or we may regard a $C^{*}(\infty)$-sum as an algebra of functions and cite Corollary 3 of Theorem 3.1. Finally, we put together Theorem 3.3, Lemma 3.2, and Corollary 3 of Theorem 3.1, and we have proved the following result.

COROLlARY. Let $B$ be an algebra satisfying the hypotheses of Theorem 3.3; then any closed right ideal in $B$ is an intersection of regular maximal right ideals.

We conclude this section with a more conventional version of the StoneWeierstrass theorem, in which we speak of continuous functions to a fixed algebra, and show that a suitable subalgebra contains all continuous func- 
tions. Theorem 3.4 is readily deduced from the two preceding theorems; one need only remark that by these theorems $B$ admits multiplication by continuous real functions, and it then becomes possible to approximate an arbitrary element of $A$ by a partition.

Theorem 3.4. Let $X$ be a locally compact Hausdorff space, $D$ a $C^{*}$-algebra which is either dual or has a unit element, and $A$ the $C^{*}$-algebra of all continuous functions vanishing at $\infty$ from $X$ to $D$. Let $B$ be a closed self-adjoint subalgebra of $A$, which contains functions taking arbitrary prescribed pairs of values in $D$ at pairs of points in $X$. Then $B=A$.

4. Hausdorff structure space. In the remainder of the paper we shall make extensive use of the structure space introduced by Jacobson [8]: the space $X$ of primitive ideals, topologized by making the closure of $\left\{P_{i}\right\}$ the set of primitive ideals containing $\cap P_{i}$. This definition always makes $X$ at least a $T_{0}$-space. We quote the following useful result from [13, Theorem 3.1].

LemMa 4.1. Let $A$ be any ring and $B$ either a two-sided ideal in $A, a$ subring of the form $e A e$, or a subring of the form $(1-e) A(1-e)$, e being an idempotent. Then there is a one-one correspondence between the primitive ideals of $B$ and those primitive ideals of $A$ not containing $B$. The mapping is implemented by $P \rightarrow P \cap B, P$ primitive in $A$, and it is a homeomorphism in the topologies of the structure spaces of $A$ and $B$.

In the context of $C^{*}$-algebras, the use of the Jacobson structure space is perhaps open to suspicion on two grounds. (1) A primitive ideal is the kernel of a purely algebraic irreducible representation. For $C^{*}$-algebras one naturally prefers to use irreducible *-representations. Since in the latter case irreducibility means the absence of closed invariant subspaces, the connection between the two concepts is not clear. However, in one direction we can clear up the ambiguity: any primitive ideal $P$ is also the kernel of an irreducible *-representation. To see this, we note that there is a regular maximal right ideal $M$ such that $P$ is the kernel of the natural representation on $A / M$. Now, in the terminology of Segal [19], there exists a state vanishing on $M[17$, p. 390]. By an appropriate application of the Krein-Milman theorem we can further get a pure state $\omega$ vanishing on $M$. The ${ }^{*}$-representation attached to $\omega$ is irreducible and has $P$ as kernel. Whether it is true conversely that the kernel of an irreducible ${ }^{*}$-representation is primitive is an open question; we shall settle a special case in the affirmative in Theorem 7.3. But in any event the structure space of Jacobson is, if anything, the smaller of the two spaces, and for many purposes this more or less justifies its use.

(2) For commutative Banach algebras, it has been found that the structure space has unsatisfactory properties, and that the right topology is the weak topology introduced by Gelfand. Of course for commutative $C^{*}$-algebras, the two are known to coincide. The next three results will indicate that 
for arbitrary $C^{*}$-algebras, the Jacobson structure space is reasonably well behaved.

Having selected the structure $X$ of primitive ideals $\left\{P_{i}\right\}$ in $A$, we may represent an arbitrary element $a$ of $A$ by the set $\left\{a_{i}\right\}$ of its images in the $C^{*}$-algebras $\left\{A / P_{i}\right\}$. We shall write $a(P)$ for the value of $a$ at the point $P$ of $X$. As observed on page 411 of [12], this functional representation preserves norm, that is, we have $\|a\|=\sup \|a(P)\|$, taken over $P$ in $X$. But in this context where $X$ is the structure space, even more is true: the sup is attained for some $P$ in $X$. Because of the identity $\left\|a^{*} a\right\|=\|a\|^{2}$, one needs to prove this only in case $a \geqq 0$, and we may assume $\|a\|=1$. To say that $\|a(P)\|$ is less than 1 is to say that the spectrum of $a(P)$ does not contain 1 , that is, that $-a$ has a quasi-inverse modulo $P$. If this is true for every $P$, then $-a$ has a quasi-inverse modulo every primitive ideal. It is known that this implies that $-a$ has a quasi-inverse in $A$ itself, contradicting $\|a\|=1$.

The following lemma will be used on several later occasions.

Lemma 4.2. Let $a$ be a self-adjoint element of a $C^{*}$-algebra with structure space $X$. Let $E$ be a closed set of real numbers containing 0 . Then the set $Z$ of $P \in X$, such that the spectrum of $a(P)$ is contained in $E$, is a closed subset of $X$.

Proof. Suppose that $Q$ is in the closure of $Z$, and $a(Q)$ has $\alpha$ in its spectrum, $\alpha \notin E$. Let $p$ be a continuous real-valued function vanishing on $E$ but not at $\alpha$. Then $p(a)$ vanishes on $Z$ but not at $Q$, contradicting the definition of the topology of $X$.

The next result indicates that it is quite generally true that the functions on the structure space "vanish at infinity."

Lemma 4.3. Let $a$ be any element of a $C^{*}$-algebra with structure space $X$, and $\epsilon$ a positive real number. Then the set $K$ of $P \in X$ for which $\|a(P)\| \geqq \epsilon$ is a compact subset of $X$.

Remark. Since $X$ is not necessarily Hausdorff, we are not asserting that $K$ is closed; indeed simple examples show that $K$ need not be closed.

Proof. Because of the identity $\left\|a^{*} a\right\|=\|a\|^{2}$, we need consider only the case where $a$ is self-adjoint. Let $\left\{F_{j}\right\}$ be a family of (relatively) closed subsets of $K$ having void intersection. We must prove that a finite subset of the $F$ 's already have void intersection. Let $I_{j}$ be the intersection of the primitive ideals comprising $F_{j}, H_{0}$ the ideal generated by the $I_{j}$ 's (in the purely algebraic sense), and $H$ the closure of $H_{0}$. We observe that $H$ is not contained in any of the primitive ideals comprising $K$. For if $H \subset R, R \in K$, then $R$ contains each $I_{j}$. By the definition of the topology of the structure space, we see that $R$ lies in each $F_{j}$, a contradiction. Next we remark that $A / H$ is semisimple (indeed a $C^{*}$-algebra). So $H$ is the intersection of the primitive ideals containing it. We write $L$ for this set of primitive ideals, and observe (as just shown) that $L$ is disjoint from $K$. Hence for every $Q$ in $L$, we have $\|a(Q)\|<\epsilon$. 
Write $r=\sup \|a(Q)\|$, taken over $Q$ in $L$. If we write $a_{1}$ for the image of $a$ mod $H$, we see that $r$ is precisely the sup of the norms of all the images of $a_{1}$ at primitive ideals of $A / H$. As we remarked above, such a sup is actually attained; in other words we have $r=\left\|a\left(Q_{0}\right)\right\|$ for a suitable $Q_{0}$ in $L$. Hence $r$ is itself less than $\epsilon$. Let $p(t)$ be a continuous real-valued function of the real variable $t$, which vanishes for $|t| \leqq r$, equals 2 for $|t| \geqq \epsilon$, and is linear between; write $b=p(a)$. Then $b$ is in $H$ since it vanishes on $L$, and $\|b(P)\|=2$ for $P$ in $K$. Since $H_{0}$ is dense in $H$, there exists an element $c$ in $H_{0}$ with $\|c-b\|$ $<1$. The element $c$ must already lie in the union of a finite number of $I$ 's, say $I_{1}, \cdots, I_{r}$. Then $F_{1} \cap \cdots \cap F_{r}$ must be void; for at any primitive ideal in this intersection $c$ would vanish, whereas $\|c(P)\| \geqq 1$ throughout $K$.

The question as to when the functional representation on the structure space gives functions with continuous norm is completely answered in the following theorem.

TheOREM 4.1. For any $C^{*}$-algebra $A$ with structure space $X$, the following statements are equivalent: (1) $X$ is Hausdorff, (2) for any $a \in A$, the function $\|a(P)\|$ is continuous on $X$.

Proof. That (2) implies (1) is immediate. If $Q, R \in X, Q \neq R$, then there exists $a \in A$ vanishing say at $Q$, but not at $R$. The continuous real-valued function $\|a(P)\|$ yields disjoint neighborhoods of $Q, R$.

To prove that (1) implies (2), we begin by investigating continuity of $\|a(P)\|$ at 0 . Take a fixed $Q \in X$, and let $I$ be the closure of the set of all $a \in A$ such that $a(P)$ vanishes in a neighborhood of $Q ; I$ is a closed two-sided ideal in $A$. We claim that $I=Q$. If not, since $I$ is the intersection of the primitive ideals containing it, $I$ will also be contained in a different primitive ideal $Q_{0}$. By the Hausdorff separation property, there exists a neighborhood $U$ of $Q$ whose closure does not contain $Q_{0}$. By the definition of the topology of $X, A$ contains an element vanishing on $U$ but not at $Q_{0}$, and this is a contradiction. At this point we know that anything vanishing at $Q$ is a limit of elements vanishing in a neighborhood of $Q$; this proves continuity of $\|a(P)\|$ at 0 .

We pass to the general proof of continuity. Because of the equation $\left\|a^{*} a\right\|=\|a\|^{2}$, it is enough to do this for self-adjoint $a$. Let $Q \in X$ and $\epsilon>0$ be given, and write $r=\|a(Q)\|$. It follows from Lemma 3.3 that in a suitable neighborhood of $Q,\|a(P)\|<r+\epsilon$. To complete the proof it will suffice to show that the set of $P$ with $\|a(P)\|>r-\epsilon$ is open; or alternatively, that the set of $P$ with $\|a(P)\| \leqq r-\epsilon$ is closed. This is a consequence of Lemma 4.2.

Remark. Putting together Lemma 4.3 and Theorem 4.1, we see that when the structure space is Hausdorff it is also locally compact. It is perhaps worth mentioning however that the structure space of a $C^{*}$-algebra is not always locally compact. A counter-example is the following: let $H$ be a Hilbert space of dimension $\boldsymbol{\aleph}_{\omega}$ and take the algebra of bounded operators on $H$ whose range has dimension less than $\boldsymbol{\aleph}_{\omega}$. It must be admitted however that 
in this example the structure space is not $T_{1}$; the author does not have an example which is $T_{1}$ but not locally compact.

In the following theorem we treat the fundamental case in which we are able to prove that the structure space is Hausdorff. The result indicates, roughly speaking, that the structure space being Hausdorff is related to the algebra being "homogeneous" in a suitable sense.

THEOREM 4.2. Let $A$ be a $C^{*}$-algebra in which for every primitive ideal $P$, $A / P$ is finite-dimensional and of order independent of $P$. Then the structure space of $A$ is Hausdorff.

Proof. We shall not prove this by a direct assault on the structure space; instead, following the idea of [12, Theorem 9.2], we provisionally introduce another space. Let $M$ be the finite-dimensional $C^{*}$-algebra (a full matrix algebra) to which each $A / P$ is isomorphic. Let $Y$ be the space of all *-homomorphisms of $A$ into $M$, including the 0 homomorphism. In the weak topology, $Y$ is a compact Hausdorff space, and the elements of $A$ are represented by continuous functions from $Y$ to $M$-these details go through more or less as usual, and we leave them to the reader. Each primitive ideal in $A$ gives rise to an orbit of points in $Y$, the orbit being in fact induced by the group $G$ of *-automorphisms of $M$. Now $G$ is compact in its natural topology, and the mapping from $G$ onto an orbit is readily seen to be continuous. Hence the orbits are closed, and we may form a well defined quotient space $X$ relative to this decomposition of $Y$. The points of $X$ are of course in one-one correspondence with the set consisting of the structure space of $A$ and a point at infinity. Being a continuous image of $Y, X$ is again compact. We can no longer speak of elements of $A$ as being represented by continuous functions $a(x)$ on $X$; but the function $\|a(y)\|$ is constant on orbits, and so gives us a uniquely defined function on $X$, which is again continuous. Moreover these functions $\|a(x)\|$ exist in sufficient abundance to separate points of $X$, for given two distinct points of $X$, we can find an element $a \in A$ vanishing at one but not at the other. From this it follows that $X$ is Hausdorff. A final remark is that the functions $\|a(x)\|$ are continuous at 1 in the sense of hypothesis $\left(a^{\prime \prime}\right)$ of Theorem 3.2. We are now ready to cite Theorem 3.2, and we arrive at the main fact we are after: $A$ contains all the real continuous functions that vanish at the 0 homomorphism. From this it would not be difficult to see directly that $X$ (with 0 deleted) is homeomorphic to the structure space; however it is faster to observe that we now know $A$ to be "central" in the sense of [12], and quote [12, Theorem 9.1].

RemarKs. 1. Another way of saying what we have just proved is that, in the homogeneous case, the hypothesis that $A$ is central in [12, Theorem 9.2 ] is redundant. We can, if we like, cite that theorem to see that the representation of $A$ on $Y$ gives rise to all continuous functions satisfying a suitable "covariance" condition. However, for the purposes of the present paper 
the representation on $X$ is decidedly more convenient, since on $X$ we get all continuous scalar functions.

2. We shall say a few words concerning how the above considerations fit into a more general framework. We stick to the homogeneous case: a Banach algebra $A$ such that all $A / P$ are isomorphic to a fixed finite-dimensional matrix algebra $M$. Without the aid of a well behaved *-operation, it seems to be difficult to construct a satisfactory theory; in general terms one may trace the trouble to the fact that the group of automorphisms of $M$ is not compact. So let us assume that $A$ has a continuous *-operation which is symmetric $\left(x^{*} x\right.$ has a quasi-inverse). Then the construction of the above space $Y$, and its reduction to $X$, go through; this appears to be a satisfactory beginning for the theory. Still, one is troubled by the fact that there are no fewer than three further plausible candidates for a space of primitive ideals: (1) the structure space, (2) primitive ideals with the weak topology induced by traces of elements, (3) the space of maximal ideals (in Gelfand's sense) of the center $Z$ of $A$. If $A$ is a $C^{*}$-algebra, all four versions can be seen to coincide, but the general situation is not clear. In particular the choice (3) is in jeopardy, since perhaps $Z=0$. The case of degree two is an honorable exception, since then $(x y-y x)^{2}$ is always in the center.

From the homogeneous case treated in Theorem 4.2, we pass on to the case where $A$ is a $C^{*}$-algebra such that each $A / P$ is finite-dimensional with a fixed upper bound on the order. This hypothesis can be described more briefly by saying that $A$ satisfies a polynomial identity. It will clarify our future considerations to make a few general remarks at this point. Consider the algebra of $n$ by $n$ matrices over a field. In the notation of [11], this satisfies the identity

$$
\left[x_{1}, \cdots, x_{r(n)}\right]=0
$$

where $r(n)$ is a certain function of $n\left({ }^{5}\right)$, and matrix algebras of higher order do not satisfy this identity. Now let $A$ be any Banach algebra; let $C_{n}$ denote the set of all primitive ideals $P$ such that $A / P$ is a $k$ by $k$ matrix algebra with $k \leqq n$, and let $I_{n}$ be the intersection of these ideals. Then $A / I_{n}$ satisfies the identity (5), and so of course does every primitive image of $A / I_{n}$. It follows that $C_{n}$ is a closed subset of the structure space $\left.{ }^{6}\right)$. Moreover it follows from Lemma 4.1 that $I_{r-1} / I_{r}$ is homogeneous: each of its primitive images is an $r$ by $r$ matrix algebra.

Let $A$ be any $C^{*}$-algebra satisfying a polynomial identity. The above defined chain of ideals $I_{n}$ reaches 0 in a finite number of steps. We have thus

(5) In a paper to appear in the Proceedings of the American Mathematical Society, Amitsur and Levitzki prove that $r(n)=2 n$. For our purposes, all that matters is that some identity shall exist that characterizes $n$ by $n$ matrices.

${ }^{(6)}$ By way of justifying the somewhat artificial introduction of polynomial identities into the discussion, it seems appropriate to remark that I see no other way to prove that $C_{n}$ is closed. 
constructed a finite composition series for $A$, with the property that every factor algebra has a Hausdorff structure space. This is the embryonic version of the more general theorems to follow in $\$ 6$.

5. A category theorem. From a certain point of view, the study of $C^{*}$ algebras may be divided into two parts: the determination of the primitive ones, and the study of how the latter combine to form a general $C^{*}$-algebra. We now pick out a class of $C^{*}$-algebras for which the first problem evaporates, and so attention is concentrated on the second.

Definition. A $C C R$-algebra is a $C^{*}$-algebra for which every primitive homomorphic image $A / P$ is isomorphic to the algebra of all completely continuous operators on a Hilbert space; more briefly, each $A / P$ is dual.

We noted in $\$ 4$ that any primitive ideal in a $C^{*}$-algebra is the kernel of an irreducible ${ }^{*}$-representation. Hence if we state that every irreducible ${ }^{*}$-representation of $A$ consists of completely continuous operators, we are uttering a hypothesis which is formally stronger than the assertion that $A$ is $C C R$. However we shall later see (Theorem 7.3) that the two hypotheses are equivalent.

In connection with Theorem 5.1, a remark is in order. It seems to be somewhat unorthodox to make use of the category concept in connection with non-Hausdorff spaces. It should be noted that compactness no longer implies second category: a countable space, in which the closed sets are finite or the whole space, is a compact $T_{1}$-space of the first category. So Theorem 5.1 tells us roughly that the structure space $X$ of a $C C R$-algebra cannot be as badly non-Hausdorff as this last example (and in fact one can deduce from Theorem 6.2 that every open subset of $X$ contains an open Hausdorff subset).

We begin with a preliminary lemma.

Lemma 5.1. Let $T_{1}, \cdots, T_{n}$ be self-adjoint completely continuous operators on a Hilbert space $H, r$ a non-negative real number, and $\alpha$ a vector of unit length in $H$ such that

$$
\left(I+T_{1}\right)^{2}+\cdots+\left(I+T_{n}\right)^{2}-r^{2} I
$$

annihilates $\alpha, I$ being the identity operator. Then for each $i$ there exists a selfadjoint completely continuous operator $U_{i}$ such that $\alpha\left(I+U_{i}\right)=0$ and $\left\|U_{i}-T_{i}\right\|$ $\leqq 3 r$.

Proof. Let $E$ be the projection on $\alpha$, and define

$$
V_{i}=E\left(I+T_{i}\right)+\left(I+T_{i}\right) E-E\left(I+T_{i}\right) E .
$$

Then $V_{i}$ is self-adjoint and completely continuous. We have

$$
\begin{aligned}
\left\|V_{i}\right\| & \leqq 3\left\|E\left(I+T_{i}\right)\right\|=3\left\|\alpha\left(I+T_{i}\right)\right\|, \\
\left\|V_{i}\right\|^{2} & \leqq 9 \sum\left(\alpha\left(I+T_{i}\right)^{2}, \alpha\right)=9 r^{2},
\end{aligned}
$$

and so $\left\|V_{i}\right\| \leqq 3 r$. We note that $\alpha V_{i}=\alpha\left(I+T_{i}\right)$. Hence the choice $U_{i}=T_{i}-V_{i}$ 
satisfies the requirements of the lemma.

TheOREM 5.1. The structure space $X$ of a CCR-algebra $A$ is of the second category.

Proof. Suppose on the contrary that $X$ is the union of an increasing sequence $C_{n}$ of closed nowhere dense sets. We proceed to define stepwise an array of objects.

(a) An increasing sequence of integers $k(1), k(2), \cdots$,

(b) For $j=2,3, \cdots$, a point (equal to a primitive ideal) $P_{j}$; we realize the algebra $A / P_{j}$ as the algebra of all completely continuous operators on a Hilbert space $H_{j}$,

(c) A vector $\alpha_{j}$ of unit norm in $H_{j}$, $\leqq j$

(d) Self-adjoint elements $a(i, j) \in A$, defined for $j=2,3, \cdots$ and $1 \leqq i$

To begin the process, we set $k(1)=1, k(2)=2$ and take $a(1,2)$ to be a self-adjoint element vanishing on $C_{1}$ and having -1 in its spectrum at a certain point $P_{2}$ not in $C_{2}$; and we pick $\alpha_{2}$ to be a vector of unit norm annihilated by the homomorphic image of $1+a(1,2) \bmod P_{2}$. (This formal use of 1 is legal in all the ensuing manipulations even if $A$ lacks a unit element.) Then take $a(2,2)$ to be a self-adjoint element vanishing on $C_{2}$ and such that $\alpha_{2}$ is also annihilated by $1+a(2,2)$ at $P_{2}$.

Now suppose the selection of $k(j), P_{j}, \alpha_{j}, a(i, j)$ has been made for $i \leqq j \leqq n$ so as to satisfy

(1) $P_{j-1}$ is not in $C_{k(j-1)}$ but is in $C_{k(j)}$,

(2) $\alpha_{j}$ is annihilated by the homomorphic image of $1+a(i, j) \bmod P_{j}$,

(3) $a(i, j)$ vanishes on $C_{k(i)}$,

(4) $a(i, j-1)$ and $a(i, j)$ coincide on $C_{k(j)}$,

(5) $\|a(i, j-1)-a(i, j)\| \leqq 2^{-i}$.

Our selections in the preceding paragraph satisfied these assumptions, insofar as they were as yet meaningful. We show how to push on to $n+1$. Take $k(n+1)$ large enough so that $P_{n}$ is in $C_{k(n+1)}$. We write

$$
b_{n}=[1+a(1, n)]^{2}+\cdots+[1+a(n, n)]^{2}-n,
$$

observing that $b_{n}$ is an actual ring element. It follows from (2) that $b_{n}$ has $-n$ in its spectrum at $P_{n}$. We claim that there exists a point $P_{n+1}$ outside $C_{k(n+1)}$, where the spectrum of $b_{n}$ contains a number arbitrarily close to $-n$; concretely, we specify that the spectrum of $b_{n}\left(P_{n+1}\right)$ shall contain a number lying within $m=2^{-2 n-8}$ of $-n$. For if not, let $p(t)$ be a continuous real-valued function which is nonzero at $-n$ and vanishes outside the open interval from $-n-m$ to $-n+m$. Then $p\left(b_{n}\right)$ vanishes in the complement of $C_{k(n+1)}$ but is different from 0 at $P_{n}$, and this contradicts the assumption that $C_{k(n+1)}$ is nowhere dense (whence its complement is dense). Hence we may choose $P_{n+1} \notin C_{k(n+1)}$ in such a way that $b_{n}\left(P_{n+1}\right)$ has in its spectrum a real number $s$ 
with $|s+n|<m$. In accordance with the above notation we realize $A / P_{n+1}$ as the algebra of all completely continuous operators on a Hilbert space $H_{n+1}$. We note that $s$ is nonzero, and consequently we may find in $H_{n+1}$ a unit vector $\alpha_{n+1}$ which is a characteristic vector for the operator $b_{n}\left(P_{n+1}\right)$, corresponding to the characteristic root $s$. Let us write $T_{i}$ for the operator representing $a(i, n)\left(P_{n+1}\right)$. Then, in view of the definition of $b_{n}$, we find that the operator

$$
\left(I+T_{1}\right)^{2}+\cdots+\left(I+T_{n}\right)^{2}-(s+n) I
$$

annihilates $\alpha_{n+1}$. We are now in a position to apply Lemma 5.1, and we deduce the existence of self-adjoint completely continuous operators $U_{1}, \cdots, U_{n}$ on $H_{n+1}$ with $\alpha_{n+1}\left(I+U_{i}\right)=0$ and $\left\|T_{i}-U_{i}\right\| \leqq 3 m^{1 / 2}<2^{-n-2}$.

Let us write $J$ for the intersection of the ideals comprising $C_{k(n+1)}$, and $K=J \cap P_{n+1}$. We note that $J$ is not contained in $P_{n+1}$, for $C_{k(n+1)}$ is a closed set not containing $P_{n+1}$. Since there are no closed ideals between $P_{n+1}$ and $A$, $J+P_{n+1}$ is dense in $A$. Hence [12, Lemma 8.1], $A / K$ is the direct sum of $A / J$ and $A / P_{n+1}$, in the $C^{*}$ as well as in the algebraic sense. It is therefore possible to find in $A / K$ a (unique) element $g_{i}$ which vanishes $\bmod J$, and maps on $U_{i}-T_{i} \bmod P_{n+1}$. We shall have $\left\|g_{i}\right\|=\left\|U_{i}-T_{i}\right\|<2^{-n-2}$. Going back to $A$, we can find a self-adjoint element $h_{i}$, mapping on $g_{i} \bmod K$, and with norm arbitrarily close to $\left\|g_{i}\right\|$; we do this so as to get $\left\|h_{i}\right\|<2^{-n-1}$.

We are ready to define $a(i, n+1)$ as $a(i, n)+h_{i}$. In view of the estimate for $\left\|h_{i}\right\|$, this satisfies condition (5) above. If we further note that $a(i, n+1)$ was constructed so as to agree with $a(i, n)$ on $C_{k(n+1)}$, and map on $U_{i}$ at $P_{n+1}$, we verify conditions (2)-(4). $P_{n+1}$ was selected so as to verify (1).

To complete this step of the induction, it now only remains to choose $a(n+1, n+1)$. For this purpose we need only choose it to be a self-adjoint element vanishing on $C_{k(n+1)}$, and such that its image mod $P_{n+1}$ annihilates $\alpha_{n+1}$. That such a selection is possible follows from the fact that (in the notation above) $A / K$ is the direct sum of $A / J$ and $A / P_{n+1}$.

Having completed the construction, we proceed as follows. From (5) we have that for each fixed $i$, the sequence $a(i, j)$ converges, say to $c_{i}$. We note two properties of the $c$ 's. Since property (3) is evidently preserved on passage to the limit, we have:

(6) $c_{i}$ vanishes on $C_{k(i)}$.

Next we deduce from (4), and the fact that the $C$ 's are an increasing sequence of sets, that $a(i, j-1)$ coincides with $a(i, s)$ on $C_{k(j)}$ for all $s \geqq j$. Now $P_{j-1}$ $\in C_{k(j)}$ by (1); it then follows from (2) that $\alpha_{j-1}$ is annihilated by the homomorphic image at $P_{j-1}$ of every $a(i, s)$ with $s \geqq j$. This is preserved in the limit, and we state:

(7) $\alpha_{j}$ is annihilated by the homomorphic image of $1+c_{i} \bmod P_{j}$.

We are now ready to exhibit the contradiction. Consider the right ideal defined by 


$$
I=\left(1+c_{1}\right) A+\cdots+\left(1+c_{n}\right) A+\cdots .
$$

Let $Q$ be any primitive ideal in $A$, say $Q \in C_{k(n)}$. Since, by (6), $c_{n}$ vanishes on $C_{k(n)}$, we have $c_{n} \in Q$. The equation $x=\left(1+c_{n}\right) x-c_{n} x$ shows that $I+Q=A$. Next, $I$ is regular, since for example $-c_{1}$ is a left unit for $I$. If $I \neq A$, then $I$ can be embedded in a regular maximal right ideal $M$. There is a primitive ideal $Q$ contained in $M$ ( $Q$ is the kernel of the natural representation of $A$ on $A / M)$, and this contradicts $I+Q=A$. Hence $I=A$, and for suitable elements $d_{i}$ :

$$
\left(1+c_{1}\right) d_{1}+\cdots+\left(1+c_{n}\right) d_{n}=-c_{1}
$$

or

$$
\left(1+c_{1}\right)\left(1+d_{1}\right)+\left(1+c_{2}\right) d_{2}+\cdots+\left(1+c_{n}\right) d_{n}=1 .
$$

This contradicts the assertion in (7) that the homomorphic images mod $P_{n}$ of $1+c_{1}, \cdots, 1+c_{n}$ all anihilate $\alpha_{n}$. This completes the proof of Theorem 5.1 .

6. Composition series. By a composition series of a $C^{*}$-algebra $A$, we mean a well-ordered ascending series of closed two-sided ideals $I_{\rho}$, beginning with 0 and ending with $A$, and such that for any limit ordinal $\lambda, I_{\lambda}$ is the closure of the union of the preceding $I$ 's. The use of an ascending series here, as opposed to a descending series, is typical of ring theory, and is analogous to the enormous superiority of minimal over maximal ideals.

THEOREM 6.1. Let $A$ be a $C^{*}$-algebra such that every primitive image $A / P$ is finite-dimensional. Then $A$ has a composition series $I_{\rho}$ such that each $I_{\rho+1} / I_{\rho}$ satisfies a polynomial identity.

Proof. In the structure space $X$ of $A$, let $C_{n}$ be the set of primitive ideals $P$ such that $A / P$ has degree not greater than $n$. Then $X$ is the union of the countable family of closed sets $C_{n}$. Since $X$ is of the second category (Theorem 5.1), one of them, say $C_{r}$, must have a nonvoid interior $U$. Let $I$ denote the intersection of the primitive ideals comprising the complement of $U$. Since the latter is closed, these are precisely the primitive ideals containing $I$; in particular we see that $I$ is nonzero. By Lemma 4.1, the primitive ideals in $I$ itself are in one-one correspondence with the members of $U$. It follows that the primitive images of $I$ are all of degree not greater than $r$. Hence $I$ satisfies a polynomial identity: to be precise, the identity for $r$ by $r$ matrices. This is the beginning of our composition series. The algebra $A / I$ again satisfies the hypothesis of our theorem (any primitive image of $A / I$ is a primitive image of $A$ ), and we continue by transfinite induction.

As we observed at the end of $\S 4$, a $C^{*}$-algebra $B$ with a polynomial identity has a (finite) composition series such that all factor algebras possess a Hausdorff structure space. In particular the first nonzero ideal in this series 
has a Hausdorff structure space, and by Lemma 4.1 the latter is homeomorphic to an open subset of the structure space of $B$. If we combine this with Theorem 6.1, and another application of Lemma 4.1, we obtain the following corollary.

Corollary. If $A$ is a $C^{*}$-algebra such that every primitive image $A / P$ is finite-dimensional, then the structure space of $A$ has a nonvoid open Hausdorff subset.

We are now able to prove our main theorem.

THEOREM 6.2. A CCR-algebra has a composition series $I_{\rho}$ such that each $I_{p+1} / I_{\rho}$ has a Hausdorff structure space.

Proof. Let $A$ be the algebra and $X$ its structure space. Select a selfadjoint element $a$ in $A$ whose spectrum lies in $(0,1)$ and actually contains 1 . At any $P \in X$, the spectrum of $a(P)$ is a finite or countable set with at most 0 as a limit point. Let $p(t)$ be a continuous real-valued function, vanishing in a neighborhood of 0 , satisfying $p(1)=1$, and, say, linear between. We pass to $b=p(a)$, and observe that every $b(P)$ has a finite spectrum lying between 0 and 1 . Let $B$ denote the intersection of the primitive ideals containing $b$, or in other words, the set of $P$ with $b(P)=0$; let $Y$ be the structure space of $B$. Lemma 4.1 shows that $B$ is again $C C R$, and that $Y$ is in a natural way an open subset of $X$. Our next efforts will be devoted to proving that $Y$ has an open Hausdorff subset.

For this purpose we first note that $b(Q) \neq 0$ for any $Q \in Y$. For $n=2,3, \cdots$, let $C_{n}$ denote the set of $Q \in Y$ for which the spectrum of $b(Q)$ lies in the closed set consisting of 0 and the closed interval from $1 / n$ to 1 . By Lemma 4.2, $C_{n}$ is closed. Also, since the spectrum of each $b(Q)$ is finite, $Y=\mathrm{U} C_{n}$. Hence (Theorem 5.1) one of the $C$ 's, say $C_{r}$, has a nonvoid interior $U$. Let $q(t)$ be a continuous real-valued function satisfying $q(0)=0, q(t)=1$ for $t \geqq 1 / r$, and write $c=q(b)$. Then at every point of $C_{r}, c$ maps into a nonzero self-adjoint idempotent, and this is a fortiori true at all the points of the closure $V$ of $U$. Let $J$ be the intersection of the primitive ideals in $B$ which comprise $V$. Let $D=B / J$; the structure space of $D$ can be identified with $V$. Let $e$ denote the homomorphic image of $c \bmod J$; then $e$ is a self-adjoint idempotent not vanishing at any point of $V$. We consider finally the algebra $e D e$; by Lemma 4.1 its structure space is again homeomorphic to $V$. Now it follows from Lemma 4.1 again that the primitive ideals in $e D e$ are of the form $R \cap e D e=e R e$, where $R$ is primitive in $D$. Thus the primitive homomorphic images of $e D e$ are of the form $e_{1}(D / R) e_{1}, e_{1}$ being the image of $e \bmod R$. We know that $D / R$ is the algebra of all completely continuous operators on a Hilbert space. It follows that $e_{1}(D / R) e_{1}$ is finitedimensional. In short, all the primitive images of $e D e$ are finite-dimensional. The corollary of Theorem 6.1 is therefore applicable, and tells us that $V$ has a 
nonvoid open Hausdorff subset, say $Z$. The intersection $T$ of $Z$ and $U$ is a nonvoid open Hausdorff subset of $Y$.

The same set $T$ is open in $X$ (since $Y$ is open in $X$ ). Let $I$ be the intersection of the primitive ideals comprising the complement of $T$. Then $I$ is a nonzero closed two-sided ideal in $A$ whose structure space is homeomorphic to the Hausdorff space $T$ (Lemma 4.1 is being used again). This is the beginning of our composition series; we continue with a similar treatment of $A / I$, and so on by transfinite induction.

7. Ideals and subrings. Our study of $C C R$-algebras now stands as follows. We have established the existence of a composition series such that all the factor algebras have a Hausdorff structure space. Next, if $A$ is a $C C R$ algebra with a Hausdorff structure space $X$, then Lemma 4.3 and Theorem 4.1 show that $X$ is locally compact, and that the representation of $A$ on $X$ gives us functions with continuous norm vanishing at $\infty$. This is precisely the setup to which Theorem 3.3 is applicable, and we are entitled to make use of that theorem and its corollary.

While this cannot be considered a complete structure theory, it does provide us with sufficient information to settle certain problems. We have selected two problems for treatment: the structure of closed one-sided ideals, and the Weierstrass-Stone theorem. The mechanism for climbing up a composition series, for the first of these problems, is provided by Lemmas 7.2 and 7.3.

Lemмa 7.1. Let $A$ be a ring, $R$ a right ideal in $A, I$ a two-sided ideal in $A$, and $N$ a regular maximal right ideal in I containing $R \cap I$. Then there exists a regular maximal right ideal $M$ in $A$, containing $R$, and such that $M \cap I=N$.

Proof. The proof is nearly identical with a portion of that of $[13$, Theorem 3.1], but we repeat it for completeness. Let $e \in I$ be a left unit modulo $N$. It cannot be the case that $A=N+N A+(1-e) A$, for then

$$
n+\sum n_{i} a_{i}+(1-e) a=e
$$

for suitable $n, n_{i} \in N, a, a_{i} \in A$. From this equation we first deduce $a \in I$ (since all other terms are in $I)$. Then $(1-e) a \in N$. A right multiplication by $e$ yields $e^{2} \in N$, a contradiction. Hence $N+N A+(1-e) A$ is proper and may be expanded to a regular maximal right ideal $M$ in $A$ with $e$ as left unit. We must verify: (1) $M \cap I=N$, (2) $M \supset R$. (1) Certainly $M \cap I$ contains $N$. If equality fails, then (since $N$ is maximal) $M \cap I=I$ and $M$ contains $e$, a contradiction. (2) If not, $M+R=A, m+r=e$ with $m \in M, r \in R$. We have $m i+r i=e i$ for any $i$ in $I$, and $m i$ and $r i$ lie in $M \cap I$ and $R \cap I$ which are both contained in $N$. Hence $e i \in N, I \subset N$, a contradiction.

LeммA 7.2. Let $A$ be a topological ring with a closed two-sided ideal I. If in $I$ and $A / I$ it is true that every closed right ideal is an intersection of regular maximal right ideals, then the same is true in $A$. 
Proof. Let $R$ be a closed right ideal in $A$, and $x$ an element lying in every regular maximal right ideal containing $R$; our problem is to prove $x \in R$. Denote images mod $I$ by primes. Then $x^{\prime}$ lies in every regular maximal right ideal containing $R^{\prime}$, and therefore by hypothesis $x^{\prime} \in R^{\prime}, x \in R+I$. Write $x=r+y, r \in R, y \in I$. Our task has been reduced to proving that $y$ is in $R \cap I$. This will be established as soon as we know that $y$ lies in every regular maximal right ideal $N$ in $I$ containing $R \cap I$. But $y$ (like $x$ ) lies in the ideal $M$ given by Lemma 7.1, and so $y$ lies in $M \cap I=N$.

Lемма 7.3. Let $A$ be a topological ring such that every $x \in A$ lies in the closure of $x A$. Suppose further that $A$ is the closure of the union of closed two-sided ideals $I_{\rho}$, in each of which it is true that every closed right ideal is an intersection of regular maximal right ideals. Then the same is true in $A$.

Proof. Let $x$ be an element lying in every regular maximal right ideal $M$ containing the closed right ideal $R$. Then $x I_{\rho} \subset M \cap I_{\rho}$. It follows from our hypothesis and Lemma 7.1 that $x I_{\rho} \subset R \cap I_{\rho} \subset R$. Hence $x A \subset R, x \in R$.

Before proceeding to use these lemmas, we remark that they allow us to handle a composition series, provided we can manage each factor algebra. It is therefore appropriate to generalize further the concept of $C C R$-algebra.

Definition. A $G C R$-algebra is a $C^{*}$-algebra having a composition series $I_{\rho}$ such that each $I_{\rho+1} / I_{\rho}$ is $C C R$.

In view of Theorem 6.2, it amounts to the same thing to assume a composition series such that each factor algebra is $C C R$ with a Hausdorff structure space. That this is really a wider class of algebras is shown by the example of the algebra of all operators of the form $\lambda I+U$, where $\lambda$ is a scalar, $I$ is the identity operator, and $U$ is completely continuous.

Theorem 7.1. Any closed right ideal in a GCR-algebra is an intersection of regular maximal right ideals.

Proof. We remarked at the beginning of this section that for a CCRalgebra with a Hausdorff structure space, the corollary of Theorem 3.3 is available. Hence Theorem 7.1 is known in that case. Lemmas 7.2 and 7.3 extend the result to an arbitrary $G C R$-algebra (concerning the hypothesis of Lemma 7.3, see footnote 3 ).

In generalizing the Weierstrass-Stone theorem we shall confine ourselves to $C C R$-algebras. In connection with Theorem 7.2, it should perhaps be remarked that this is not the only possible form for the Weierstrass-Stone theorem to take-one might for example have the closed subalgebra separate suitable two-sided ideals, or pure states. However, it can be seen that for $C C R$-algebras these alternative versions come to essentially the same thing.

THEOREM 7.2. Let $A$ be a CCR-algebra and $B$ a closed self-adjoint subalgebra with the following property: for any distinct regular maximal right ideals in $A, B$ contains an element in one but not in the other. Then $B=A$. 
Proof. Let $P, Q$ be any distinct primitive ideals in $A$. It follows from [12, Lemma 8.1] that the algebra $A_{1}=A /(P \cap Q)$ is the direct sum of $A / P$ and $A / Q$, that is, $A_{1}$ is the direct sum of two simple dual $C^{*}$-algebras. The algebra $B_{1}=B /(B \cap P \cap Q)$ is in a natural way embedded in $A_{1}$, and $B_{1}$ separates the regular maximal right ideals of $A_{1}$ in the same sense that $B$ does so in $A$ (the simple argument for this was provided in the proof of Theorem 2.2). It follows from Theorem 2.2 that $B_{1}=A_{1}$; in other words, $B$ contains elements taking arbitrary pairs of values at pairs of primitive ideals in $A$.

Now let $\left\{I_{\rho}\right\}$ be a composition series for $A$ such that each $I_{\rho+1} / I_{\rho}$ is $C C R$ with a Hausdorff structure space. Let us for brevity write $I$ for the first nonzero ideal of the series; $I$ is thus itself a $C C R$-algebra with Hausdorff structure space. Let $R$ be any primitive ideal in $I$, and $e$ any self-adjoint idempotent in $I / R$. The next few paragraphs will be devoted to proving that $B \cap I$ contains an element mapping on $e \bmod R$.

We first consider an arbitrary $I_{\rho}$ and observe (by Lemma 4.1) that there exists a unique primitive ideal $R_{\rho}$ in $I_{\rho}$ such that $R_{\rho} \cap I=R$. In the present context where all factor algebras modulo a primitive ideal are simple, the two algebras $I / R$ and $I_{\rho} / R_{\rho}$ are necessarily isomorphic. There will be no ambiguity if we use the same letter $e$ to denote the element in $I_{\rho} / R_{\rho}$ which corresponds to $e$ in $I / R$.

Suppose we have obtained a self-adjoint element $x$ in $B \cap I_{\lambda}$ such that $x\left(R_{\lambda}\right)=e$. We shall show how to achieve this with a smaller ordinal than $\lambda$

Case I. $\lambda$ a limit ordinal. We note that $I_{\lambda}$ is the closure of the union of $I_{\mu}$ with $\mu<\lambda$. Hence for a suitable $I_{\mu}$ we have an element $y \in I_{\mu}$ with $\|y-x\|$ $<1 / 2$. In other words, the image of $x$ in $I_{\lambda} / I_{\mu}$ is an element of norm less than $1 / 2$. Let $p(t)$ be a continuous real-valued function vanishing for $|t| \leqq 1 / 2$, and satisfying $p(1)=1$. Then $p(x)$ is in $B \cap I_{\mu}$, and $p(x)$ is again equal to $e$ at $R_{\mu}$.

Case II. $\lambda$ not a limit ordinal. The algebra $I_{\lambda} / I_{\lambda-1}$ has a structure space $Y$ which is locally compact Hausdorff, and the elements of $I_{\lambda} / I_{\lambda-1}$ are represented by functions with continuous norm vanishing at $\infty$. It will be convenient to think of $Y$ as consisting of the primitive ideals of $I_{\lambda}$ which contain $I_{\lambda-1}$. Among these we do not find $R_{\lambda}$ (unless $I_{\lambda}=I$, in which case the present paragraph is superfluous). Let $S$ be the general point of $Y$, and let us write $S^{\prime}$ for the unique primitive ideal in $A$ with $S^{\prime} \cap I_{\lambda}=S$; similarly write $R^{\prime}$ for the unique expansion of $R$ to a primitive ideal in $A$. Then by the opening paragraph of the proof, we know that $B$ contains an element $y$ with $y\left(S^{\prime}\right)=0$, $y\left(R^{\prime}\right)=e$. We pass to the element $z=y^{*} x^{*} x y$ which is self-adjoint, lies in $B \cap I_{\lambda}$, and enjoys the properties $z(S)=0, z\left(R_{\lambda}\right)=e$. Let $q$ be a continuous real-valued function vanishing in a neighborhood of 0 and satisfying $q(1)=1$. Then when we take $w=q(z)$, we achieve the following: $w \in B \cap I_{\lambda}, w\left(R_{\lambda}\right)=e$, $w$ vanishes in a neighborhood of $S$, and further vanishes outside a compact set $K \subset Y$. For any point in $K$ we can similarly get hold of an element in $B \cap I_{\lambda}$ which vanishes in a neighborhood of that point, and takes the value $e$ 
at $R_{\lambda}$. By a finite number of such elements we can cover all of $K$. Let $v$ be the result of multiplying $w$ by these elements; then $v$ vanishes on $Y$ and so lies in $I_{\lambda-1}$. If we finally take $v^{*} v$ we have found the desired self-adjoint element in $B \cap I_{\lambda-1}$, mapping on $e$ at $R_{\lambda-1}$.

Now to start the process going we pick any self-adjoint element $x_{0}$ in $B$ with $x_{0}\left(R^{\prime}\right)=e$. We apply the result just obtained, first with $I_{\lambda}=A$, then with $I_{\lambda}$ the next ideal we reach, and so forth. This gives us a chain of elements $x_{0}, x_{1}, \cdots$ which must terminate in a finite number of steps, for we are descending a well ordered set. The last element $x_{k}$ of the chain lies in $B \cap I$ and satisfies $x_{k}(R)=e$, as desired.

We can now complete the proof of the theorem. Let $P, Q$ be distinct primitive ideals in $I$. Let $e, f$ be self-adjoint idempotents in $I / P, I / Q$. Following the same notation as above, we write $P^{\prime}, Q^{\prime}$ for the extensions of $P$ and $Q$ to primitive ideals in $A$, and we continue to write $e$ and $f$ for the corresponding elements in $A / P^{\prime}, A / Q^{\prime}$. By the first paragraph of the proof, we know that $B$ contains elements $y_{1}, y_{2}$ with $y_{1}(P)=e, y_{2}(Q)=f, y_{1}(Q)=y_{2}(P)$ $=0$. We have further just proved that $B \cap I$ contains $y_{3}, y_{4}$ with $y_{3}(P)=e$, $y_{4}(Q)=f$. Then $y_{1} y_{3}+y_{2} y_{4}$ lies in $B \cap I$ and maps on $e, f$ at $P, Q$. Translated, this says that the algebra $B^{\prime}=(B \cap I) /(B \cap P \cap Q)$ contains all the self-adjoint idempotents of $I /(P \cap Q)$ (observe that the former algebra is naturally embedded in the latter). But the algebra $I /(P \cap Q)$ is dual, and the self-adjoint idempotents of any dual $C^{*}$-algebra generate a dense subalgebra. By Lemma $2.1, B^{\prime}$ is all of $I /(P \cap Q)$. In other words, $B \cap I$ contains elements taking arbitrary pairs of values at $P$ and $Q$. Since $I$ is $C C R$ with a Hausdorff structure space, we are in precisely the situation to which Theorem 3.3 is applicable (compare the opening remarks of this section). We deduce that $B \cap I$ is closed under multiplication by any continuous real-valued function on the structure space of $I$. A partition argument now allows us to approximate elements of $I$ arbitrarily closely by elements of $B \cap I$; this is done exactly as in Theorem 3.1, and we leave the details to the reader. Hence $B \cap I=I$, that is $B$ contains $I$.

Suppose we have reached the statement $B \supset I_{\rho}$ for a certain ordinal $\rho$. The algebra $B /\left(B \cap I_{\rho}\right)$ satisfies the hypothesis of our theorem relative to $A / I_{\rho}$, that is, $B /\left(B \cap I_{\rho}\right)$ separates the regular maximal right ideals of $A / I_{\rho}$. We may therefore apply the result just proved, and we have $B \supset I_{\rho+1}$. By a transfinite induction, $B=A$. This completes the proof of Theorem 7.2.

REMARK. It seems to be a plausible conjecture that Theorems 7.1 and 7.2 are actually valid for any $C^{*}$-algebra.

We shall next briefly discuss the *-representations of a $G C R$-algebra, confining our discussion to factor representations. A *-representation of a $C^{*}$-algebra is said to be a factor representation if the weak closure of the set of representing operators is a factor in the sense of Murray and von Neumann [14]. (The Murray-Neumann theory is formally available only in the sepa- 
rable case. However, the sole facts we shall need are easily supplied in the nonseparable case, and are as follows: (1) a factor has no ideal divisors of 0 , in the sense of Lemma 2.5. The same condition is called quasi-transitivity by Rickart [18]. (2) If a factor has a minimal right ideal, then it is isomorphic to the algebra of all bounded operators on a Hilbert space. One might call this "type $I_{\aleph}$," where $\boldsymbol{\aleph}$ is the dimension of the Hilbert space, but we shall simply refer to it as type I.)

Lemma 7.4. A GCR-algebra with no ideal divisors of 0 is primitive with a minimal right ideal.

Proof. Suppose first that $A$ is a $C C R$-algebra with a Hausdorff structure space $X$. If $X$ contains more than a single point, take two disjoint open sets, and form the ideals $I, J$ of all functions vanishing on their respective complements. Then $I, J$ are nonzero but $I J=0$. Hence $A$ must be primitive.

In the general case where $A$ is $G C R$, there will exist a closed ideal $I_{1}$ (the beginning of a composition series for $A$ ) such that $I_{1}$ is a $C C R$-algebra with a Hausdorff structure space. As a ring on its own merits, $I_{1}$ has no ideal divisors of 0 (any closed ideal in $I_{1}$ is an ideal in $A$ ). Hence $I_{1}$ is primitive. Let $e$ be a primitive idempotent in $I_{1}$. Then $e I_{1}=e A$ is a minimal right ideal in $A$, and the fact that there are no ideal divisors of 0 implies that the natural representation of $A$ on $e A$ is faithful. Hence $A$ is primitive with a minimal right ideal.

An immediate corollary of this lemma, in view of the remarks above, is the following theorem.

THEOREM 7.3. Any factor representation of a GCR-algebra is of type $\mathbf{I}$, and the kernel of the representation is a primitive ideal.

Theorem 7.3 is in particular applicable to irreducible ${ }^{*}$-representations, since they are factor representations. In the case of $C C R$-algebras, this tells us that all irreducible *-representations of a $C C R$-algebra consist of completely continuous operators, and we have thus cleared up the question discussed at the beginning of $\S 5$. We use this fact in proving the next theorem.

THEOREM 7.4. If $A$ is a CCR-algebra (resp. GCR-algebra), so is any homomorphic image or closed self-adjoint subalgebra of $A$.

Proof. (1) If $A$ is $C C R$, then so is any homomorphic image $B$ of $A$ (by a homomorphic image we mean the $C^{*}$-algebra obtained in canonical fashion by reducing $A$ modulo a closed two-sided ideal); this is immediate from the definition of $C C R$ and the fact that the primitive images of $B$ are also primitive images of $A$.

(2) Let $A$ be $C C R$ and $B$ a closed self-adjoint subalgebra. To prove that $B$ is $C C R$ it will suffice (in fact by Theorem 7.3 it is equivalent) to prove that any irreducible ${ }^{*}$-representation of $B$ consists of completely continuous 
operators. Suppose then we have an irreducible *-representation $\theta$ of $B$ on a Hilbert space $H$. It is known [15] that $\theta$ can be extended to $A$ in the following sense: there is an irreducible *-representation $\phi$ of $A$ on a Hilbert space $K$, and when $\phi$ is specialized to $B$ there is a closed invariant subspace on which $\phi$ is unitarily equivalent to $\theta$. Since $\phi$ sends $A$ into completely continuous operators, $\theta$ does the same to $B$.

(3) Let $A$ be $G C R$ and $I_{\rho}$ a composition series with each $I_{\rho+1} / I_{\rho} C C R$. Suppose that for all ordinals $\rho$ less than $\lambda$, we know that the homomorphic images of $I_{\rho}$ are $G C R$, and let $J$ be a closed ideal contained in $I_{\lambda}$. Write $K_{\rho}=\left(J+I_{\rho+1}\right) /\left(J+I_{\rho}\right) ; K_{\rho}$ is isomorphic to $I_{\rho+1} /\left[I_{\rho+1} \cap\left(J+I_{\rho}\right)\right]$. For $\rho+1$ $<\lambda, K_{\rho}$ is $G C R$ by our inductive assumption, while if $\lambda$ is not a limit ordinal, $K_{\lambda-1}=I_{\lambda} /\left(J+I_{\lambda-1}\right)$ is $C C R$, being a homomorphic image of the $C C R$-algebra $I_{\lambda} / I_{\lambda-1}$. We have thus verified that $\left\{J+I_{\rho} ; \rho \leqq \lambda\right\}$ is a composition series running from $J$ to $I_{\lambda}$, with all quotient algebras $G C R$. We interpolate further composition series into each of these $G C R$-algebras, and we have shown that $I_{\lambda} / J$ is $G C R$.

(4) Again let $A$ be $G C R$ with composition series $I_{\rho}$, and let $B$ be a closed self-adjoint subalgebra. The algebra $\left(B \cap I_{\rho+1}\right) /\left(B \cap I_{\rho}\right)$ is $C C R$, being in a natural way a subalgebra of $I_{\rho+1} / I_{\rho}$. We shall know that $B$ is $G C R$ as soon as we verify that $\left\{B \cap I_{\rho}\right\}$ is indeed a composition series, that is, for a limit ordinal, we must show that $B \cap I_{\lambda}$ is the closure of the union of the preceding $B \cap I_{\rho}$. So let $x$ be a self-adjoint element in $B \cap I_{\lambda}$. For any $\epsilon>0$ there exists $\mu<\lambda$ and a self-adjoint $y$ in $I_{\mu}$, such that $\|x-y\|<\epsilon / 2$. Let $f(t)$ be a continuous real-valued function with $f(t)=0$ for $|t| \leqq \epsilon / 2, f(t)=t$ for $|t| \geqq \epsilon$, and linear between. Then $f(x) \in B \cap I_{\mu}$ and $\|f(x)-x\|<\epsilon$. This completes the proof of Theorem 7.4.

The next theorem indicates, roughly speaking, the limits of our theory when applied to a general $C^{*}$-algebra; we have extensive information on $K$, but can say nothing about $A / K$.

Theorem 7.5. In any $C^{*}$-algebra $A$ there exists a unique maximal GCRideal $K$, and $A / K$ has no nonzero $G C R$-ideals.

Proof. (By a $G C R$-ideal we mean a closed two-sided ideal which is itself a $G C R$-algebra.) Only two things need verification: (1) the union of two $G C R$-ideals is a $G C R$-ideal, (2) the union of a chain of $G C R$-ideals is a $G C R$ ideal. (1) If $I, J$ are $G C R$, then $(I+J) / J \cong I /(I \cap J)$ is $G C R$ by Theorem 7.4. We may then run a $C C R$ composition series from 0 to $J$ and thence to $I+J$. (2) We may assume that the chain $\left\{I_{\rho}\right\}$ is well ordered. By Theorem 7.4 each $I_{\rho+1} / I_{\rho}$ is $G C R$, and by interpolation we can build a composition series from 0 to $U I_{\rho}$.

As further results one might mention that the algebra of continuous functions from a compact Hausdorff space to a $G C R$-algebra is $G C R$, and that the $C^{*}(\infty)$-sum of $G C R$-algebras is $G C R$. In this last result one cannot replace 
the $C^{*}(\infty)$-sum by the $C^{*}$-sum (the $C^{*}$-sum is the algebra of all bounded sequences). In fact we prove a counter-theorem.

Theorem 7.6. If the $C^{*}$-sum of $C^{*}$-algebras $\left\{A_{i}\right\}$ is $G C R$, then all but a finite number of the A's satisfy a fixed polynomial identity.

Proof. Suppose the contrary. Then we can find a sequence of $A$ 's, say $B_{1}, B_{2}, \cdots$, such that $B_{n}$ does not satisfy the polynomial identity for $n$ by $n$ matrices. For at least one primitive ideal $P_{n}$ in $B_{n}$, it will likewise be the case that $B_{n} / P_{n}$ fails to satisfy this identity. We write $C_{n}=B_{n} / P_{n}, C$ for the $C^{*}$-sum of $\left\{C_{n}\right\}$, and observe that there is a natural homomorphism of the $C^{*}$-sum of $\left\{A_{i}\right\}$ onto $C$. By Theorem 7.4, $C$ is $G C R$. Next we note that $C_{n}$ is primitive, and that a primitive ring has no ideal divisors of 0 [7, Lemma 4]. It follows from Lemma 7.4 that $C_{n}$ has a minimal right ideal. Now there are two possibilities: $C_{n}$ may be finite-dimensional, in which case it has degree greater than $n$. Otherwise, we cite [12, Theorem 7.3] to deduce that $C_{n}$ contains all the completely continuous operators on an infinite-dimensional Hilbert space. The latter algebra contains subalgebras isomorphic to total matrix algebras of arbitrarily high degree. So in either case we have shown that $C_{n}$ contains a subalgebra isomorphic to $M_{n}$, the $n$ by $n$ total matrix algebra. The $C^{*}$-sum of the algebras $\left\{M_{n}\right\}$ is thus a closed self-adjoint subalgebra of $C$, and by Theorem 7.4 is $G C R$. The proof will therefore be complete when we establish the following lemma.

Lemma 7.5. Let $M_{n}(n=1,2, \cdots)$ be the $n$ by $n$ total matrix $C^{*}$-algebra, and $A$ the $C^{*}$-sum of $\left\{M_{n}\right\}$. Then $A$ is not a GCR-algebra.

Proof. Let $I$ be any maximal ideal in $A$. If $A$ is $G C R$, so is $A / I$ (Theorem 7.4). Also $A / I$ is simple with a unit element (since $A$ has a unit), certainly has no ideal divisors of 0 , and so by Lemma 7.4 has a minimal right ideal. Theorem 7.3 of [12] is applicable, and shows that $A / I$ must be finite-dimensional, for otherwise it would have a proper two-sided ideal (the ideal of completely continuous operators, for example).

On the other hand, let $T$ be the $C^{*}(\infty)$-sum of the $M^{\prime}$ 's, that is, the set of sequences vanishing at $\infty ; T$ is a closed ideal in $A$. Since $A$ has a unit, $T$ can be enlarged to at least one maximal ideal. In contradiction to the preceding paragraph, we shall show that $A / I$ is infinite-dimensional for any maximal ideal $I$ containing $T$.

For suppose on the contrary that $A / I$ is an $r$ by $r$ total matrix algebra. Consider an idempotent $e$ in $A, e=\left\{e_{n}\right\}$, where $e_{n}$ is a projection on a $k_{n}$ dimensional subspace. We assert the following: if for sufficiently large $n$ (say $n \geqq N$ ) we have $k_{n}(r+1) \leqq n$, then $e \in I$. Since the unit element of $A$ is evidently the sum of a finite number of such idempotents, this will complete the proof. For $n \geqq N$, it is possible to find in $M_{n}, r$ further idempotents to go along with $e_{n}$, all of them projections on $k_{n}$-dimensional subspaces, and any 
two of them orthogonal. Say we pick $e_{n}(i), i=1, \cdots, r+1$, where $e_{n}(1)=e_{n}$. Define $e(i)$ to be the element in $A$ whose $n$th coordinate is $e_{n}(i)$; it does not matter what we do with the coordinates before $N$. Then $e(1), \cdots, e(r+1)$ map into orthogonal idempotents in $A / I$, and one of them must be 0 , say $e(i)$. But $e(1)$ can be expressed in the form $e(1)=f e(i) g$, and so $e(1)=e$ is in $I$.

8. Regular ideals. Ideal theory in rings without a unit element is complicated by the possibility that maximal right ideals, or even closed maximal right ideals, may be nonregular. We shall now indicate that, to some extent, this does not happen in the $C^{*}$-algebras under discussion.

Lemma 8.1. Let $A$ be a ring with a two-sided ideal I such that $A I=I$. Suppose that all maximal right ideals in $I$ are regular, and that $M$ is a maximal right ideal in $A$ not containing $I$. Then $M$ is regular.

Proof. We assert that $N=M \cap I$ is maximal in $I$. For let $x \in I, x \notin N$. For any $a$ in $A$ there exist $b \in A, m \in M$, and an integer $p$ such that $x(b+p)$ $+m=a$. A right multiplication by $I$ shows that $x I+N \supset A I=I$, as desired. So $N$ is maximal and hence regular, say with left unit $e \in I$. By Lemma 7.1 there exists a regular maximal right ideal $M_{1}$ in $A$ with $M_{1} \cap I=N$. If $M_{1} \neq M$, then $M+M_{1}=A$, and we may write $m^{\prime}+m_{1}=e, m^{\prime} \in M, m_{1} \in M_{1}$. A right multiplication by $I$ shows $e I \subset N$, whence $I \subset N$, a contradiction. Hence $M=M_{1}$ is regular $\left({ }^{7}\right)$.

Lemma 8.2. Let $A$ be a ring with a two-sided ideal $I$ such that $A I=I$. If in $I$ and in $A / I$ all maximal right ideals are regular, then the same is true in $A$.

Proof. Let $M$ be a maximal right ideal in $A$. If $M \supset I$ the result is clear, and otherwise we cite Lemma 8.1.

Lemma 8.3. Let $A$ be a Banach algebra with $A^{2}=A$ which is the closure of the union of two-sided ideals $\left\{I_{r}\right\}$ satisfying $A I_{r}=I_{r}$. Suppose that every maximal right ideal in each $I_{r}$ is regular. Then the same is true in $A$.

Proof. Let $M$ be a maximal right ideal in $A$. If $M$ fails to contain some $I_{r}$, we cite Lemma 8.1. So assume $M \supset \bigcup I_{r}$. Let $P$ be the primitive ideal attached to $M$ : the set of all $x$ in $A$ with $A x \subset M$. Then $P$ contains $U I_{r}$. In a Banach algebra any primitive ideal is closed, and so $P=A, A^{2} \subset M$, a contradiction.

Lemma 8.4. Let $A$ be a ring with center $Z$. Suppose that every element of $A$ is a multiple of some element of $Z$, and that for any primitive ideal $P, A / P$ has a unit element. Then any maximal right ideal $M$ in $A$ is regular.

Proof. Let $P$ be the primitive ideal attached to $M$ : the set of all $x$ in $A$ with $A x \subset M$. It will suffice to prove $P \subset M$. Let $x \in P$ and write $x=y z$, $z \in Z$. If $y \in M$, we are finished. Otherwise any $a$ in $A$ can be written $a$

( ${ }^{7}$ This argument in effect proves that the ideal $M$ of Lemma 7.1 is unique. 
$=y(b+n)+m$ for $b \in A, m \in M$, and $n$ an integer. A multiplication by $z z_{1}$, where $z_{1}$ is a general element of $Z$, shows that $A z \subset M$. Now suppose $z \notin M$. Then (with a similar notation) we have $a_{1}=z\left(b_{1}+n_{1}\right)+m_{1}$, and multiplication by the general element of $Z$ gives $M \supset A Z=A$, a contradiction. Hence $z \in M$ and $x \in M$, as desired.

This sequence of lemmas can be applied as follows. First let $A$ be a $C^{*}$ algebra for which each primitive image is finite-dimensional of a fixed degree, and let $Z$ be its center. Then we may deduce from Theorem 4.2 that the hypothesis of Lemma 8.4 is satisfied; in fact, if $a \in A$ has the functional representation $f(x)$ on the structure space of $A$, then $z=\|f(x)\|^{1 / 2}$ is in $Z$ and $a$ is a multiple of $z\left({ }^{8}\right)$. So we know that the maximal right ideals of $A$ are regular. Then by applying Theorem 6.1 and Lemmas 8.2 and 8.3, we obtain the following theorem. (It is to be observed that the hypotheses of these lemmas are fulfilled since any $C^{*}$-algebra is equal to its square.)

THEOREM 8.1. If $A$ is a $C^{*}$-algebra for which every primitive image is finitedimensional, then the maximal right ideals of $A$ are regular.

We conclude with two similar results for which we omit the proofs. (1) For two-sided ideals an analogue of Lemma 8.3 seems not to be available. However the analogues of Lemmas 8.2 and 8.4 hold and we find: in a $C^{*}$ algebra with a polynomial identity all maximal two-sided ideals are regular. (2) When we push on to $G C R$-algebras, we are impeded by a lack of information about maximal ideals in the algebra of completely continuous operators on Hilbert space. However closed ideals are easily treated and we have: the closed maximal right ideals in a GCR-algebra are regular.

9. Weakly closed algebras. There is virtually no intersection between the Murray-von Neumann theory of rings of operators and the results of the present paper. In fact, the $G C R$ hypothesis is so restrictive in the context of weakly closed operator algebras that the algebras become trivial.

TheOREM 9.1. A weakly closed self-adjoint algebra of operators on Hilbert space is GCR if and only if it satisfies a polynomial identity, and it is then the direct sum of a finite number of algebras, each of which is a full matrix algebra over a commutative algebra.

Theorem 9.1 can (at least in the separable case) be deduced from von Neumann's reduction theory [16]; it is also possible to give a brief direct proof. We omit the details.

10. Algebraic algebras. There appears to be a rather far-reaching analogy between algebraic algebras and $C^{*}$-algebras. In the commutative case this analogy was explored in [2]. In the more general case of a polynomial identity, there is a striking similarity between Theorem 4.2 of this paper and [13,

$\left(^{8}\right)$ That $A$ does actually contain an element $b$ with $a=b z$ can be seen by approximating $b$ locally, and combining the parts by a partition. 
Theorem 4.1], each of these theorems asserting that for a homogeneous algebra the structure space is Hausdorff. In-this section we shall show that the main device of the present paper-the category argument-also has a perfect counterpart in algebraic algebras, and we shall derive some corollaries concerning Kurosch's problem.

We shall make free use of the results in [2] and [13], but for the reader's convenience we collect the relevant definitions. A ring is biregular if every principal two-sided ideal is generated by a central idempotent, strongly regular if for every $a$ there exists an $x$ with $a^{2} x=a, \pi$-regular if for every $a$ there exists an $x$ and an integer $n$ with $a^{n} x a^{n}=a^{n}$. An algebra is algebraic if every element satisfies a polynomial equation, locally finite if every finitely generated subalgebra is finite-dimensional. Any algebraic algebra is $\pi$-regular; any strongly regular ring is both $\pi$-regular and biregular. Kurosch's problem is to determine whether every algebraic algebra is locally finite.

We first exhibit the category argument in a context which is simplified by the fact that we already know the structure space is Hausdorff. We use the term "composition series" as above; $I_{\rho}$ is a composition series for $A$ if it is a well ordered ascending chain of ideals running from 0 to $A$, such that for any limit ordinal $\lambda, I_{\lambda}$ is the union of the preceding $I$ 's.

THEOREM 10.1. Let $A$ be a biregular ring such that every primitive image $A / P$ satisfies a polynomial identity (or equivalently is finite-dimensional over its center). Then $A$ has a composition series $I_{\rho}$ such that each $I_{\rho+1} / I_{\rho}$ satisfies a polynomial identity.

Proof. Let $C_{n}$ be the set of all primitive ideals $P$ such that $A / P$ satisfies the identity for $n$ by $n$ matrices. Then $C_{n}$ is a closed subset of the structure space $X$, and $X=\cup C_{n}$. Since $X$ is locally compact Hausdorff [2, Theorem 2.2], it is of the second category. Hence some $C_{r}$ contains a nonvoid open set $U$. (We could take $U$ open and closed and get a direct summand if we wished.) Let $I$ be the intersection of the ideals comprising the complement of $U$. Then $I$ satisfies the identity for $r$ by $r$ matrices; it is the beginning of our composition series, we continue on $A / I$, and so on by transfinite induction.

We now prove the analogue of Theorem 5.1. It is typical that the proof here is simpler: the elaborate construction of Theorem 5.1 is replaced by some simple juggling of idempotents.

THEOREM 10.2. The structure space of any $\pi$-regular ring is of the second category.

Proof. Suppose on the contrary that the structure space $X$ is the union of the ascending sequence $C_{n}$ of closed nowhere dense sets. We proceed to construct a sequence $e_{1}, e_{2}, \cdots$ of nonzero idempotents satisfying: (1) $e_{n}$ vanishes on $C_{n}$, (2) $e_{n} \in e_{n-1} A e_{n-1}$. Suppose this has been done as far as $e_{r}$. We note that the complement of $C_{r+1}$ is dense (since $C_{r+1}$ is nowhere dense). 
It cannot be the case that the nonzero element $e_{r}$ vanishes everywhere on the complement of $C_{r+1}$. Hence there exists $P \notin C_{r+1}$ such that $e_{r}(P) \neq 0$. Write $J$ for the intersection of the ideals comprising $C_{r+1}$. We observe that $J$ is not contained in $P$, for $C_{r+1}$ is closed and does not contain $P$. If we can find a nonzero idempotent in $e_{r} J e_{r}$, this will be a suitable choice for $e_{r+1}$. Let $x$ be any element in $e_{r} J e_{r}$. We have $x^{s} y x^{s}=x^{s}$ for suitable $s$ and $y$. Then $x^{8} y e_{r}$ is an idempotent in $e_{r} J e_{r}$; if it is zero, so is $x^{s}=\left(x^{8} y e_{r}\right) x^{s}$. Thus the absence of nonzero idempotents in $e_{r} J e_{r}$ means that it is a nil ring. Then $e_{r} J$ is a nil right ideal in $A$, hence contained in the radical of $A$, hence contained in $P$. Likewise $\left(A e_{r} A\right) J\left(A e_{r} A\right)$ is contained in $P$. By [7, Lemma 4] either $J$ or $A e_{r} A$ is in $P$, and both possibilities are excluded. Thus the construction of the $e$ 's can be successfully continued.

Let $I$ be the right ideal in $A$ defined by

$$
I=\left(1-e_{1}\right) A+\left(1-e_{2}\right) A+\cdots+\left(1-e_{n}\right) A+\cdots .
$$

The proof that $I=A$ is identical with the corresponding portion of the proof of Theorem 5.1. Hence for suitable $n$ and elements $a_{i}$ :

$$
\left(1-e_{1}\right) a_{1}+\left(1-e_{2}\right) a_{2}+\cdots+\left(1-e_{n}\right) a_{n}=e_{1} .
$$

We observe that $e_{n} e_{i}=e_{n}$ for $i \leqq n$, and hence a left-multiplication by $e_{n}$ yields $e_{n}=0$, a contradiction.

Theorem 10.2 could be used for a study of ideals and subrings, along the lines of $\$ 7$. However we shall here confine ourselves to deriving some consequences concerning Kurosch's problem.

THEOREM 10.3. Let $A$ be a semi-simple algebraic algebra with the property that every primitive image satisfies a polynomial identity. Then $A$ is locally finite.

Proof. The structure space $X$ of $A$ is of the second category by Theorem 10.2. Exactly as in the proof of Theorem 10.1 we find in $X$ an open set $U$ such that for $P \in U, A / P$ satisfies a fixed polynomial identity, say the one for $r$ by $r$ matrices; and we let $I$ denote the intersection of the ideals comprising the complement of $U$. Since $A$ is semi-simple, so is $I$. Moreover every primitive image of $I$ corresponds to an $A / P$ with $P \in U$; hence $I$ itself satisfies the identity for $r$ by $r$ matrices. The algebra $A / I$ again satisfies the hypotheses of our theorem, and the process can be continued. In this way we build a composition series for $A$, say $I_{\rho}$, with each $I_{\rho+1} / I_{\rho}$ satisfying a polynomial identity. Suppose that for all $\rho$ less than a certain ordinal $\lambda$ we have proved that $I_{\rho}$ is locally finite. If $\lambda$ is a limit ordinal, it is clear that $I_{\lambda}$ is again locally finite; indeed any finitely generated subalgebra of $I$ already lies in some $I_{\rho}$. If $\lambda$ is not a limit ordinal, we quote [13, Theorem 6.1] which asserts that any algebraic algebra satisfying a polynomial identity is locally finite. Thus $I_{\lambda} / I_{\lambda-1}$ is locally finite, $I_{\lambda-1}$ is locally finite by induction, and hence $I_{\lambda}$ is locally finite by $[9$, Theorem 15]. 
THEOREM 10.4. If Kurosch's problem has an affirmative answer for nil algebras and for primitive algebraic algebras, then it has an affirmative answer for all algebraic algebras.

Proof. Let $A$ be a finitely generated algebraic algebra. Our task is to prove $A$ finite-dimensional, granted the above hypotheses. The radical $R$ of $A$ is a nil algebra, and so by hypothesis locally finite. Next we observe that every primitive image of $A / R$ is (like $A$ ) finitely generated, and hence finitedimensional by hypothesis. This makes Theorem 10.3 applicable, and we deduce that $A / R$ is locally finite (and indeed finite-dimensional since it is finitely generated). Now that we know that both $R$ and $A / R$ are locally finite, we quote [9, Theorem 15] to conclude the proof. (We remark that the proof has actually used a slightly weaker hypothesis; we needed only that a finitely generated primitive algebra is finite-dimensional).

Our final theorem gives another conditional result of this kind.

THEOREM 10.5. If Kurosch's problem has an affirmative answer for all algebraic division algebras, then it has an affirmative answer for all algebraic algebras of bounded index.

Proof. It was observed after [13, Theorem 6.1] that to solve Kurosch's problem for algebraic algebras of bounded index, it would suffice to treat the case of index 0 (that is, no nilpotent elements). Such an algebra is strongly regular, and so is any subalgebra. Thus we have reduced the problem to the case of a finitely generated algebraic algebra $A$ without nilpotent elements. The primitive images of $A$ are division algebras, are again finitely generated, and consequently are finite-dimensional by hypothesis. Quotation of Theorem 10.3 completes the proof (actually the more elementary Theorem 10.1 would suffice).

\section{BIBLIOGRAPHY}

1. A. A. Albert, Structure of algebras, Amer. Math. Soc. Colloquium Publications, vol. 24, New York, 1939.

2. R. Arens and I. Kaplansky, Topological representation of algebras, Trans. Amer. Math. Soc. vol. 63 (1948) pp. 457-481.

3. N. Bourbaki, Élements de mathématique, vol. 3, Topologie gênérale, Actualités Scientifiques et Industrielles, no. 1045, Paris, 1948.

4. I. Gelfand and M. Neumark, On the imbedding of normed rings into the ring of operators in Hilbert space, Rec. Math. (Mat. Sbornik) N. S. vol. 12 (1943) pp. 197-213.

5. - Unitary representations of the Lorentz group, Izvestia Akademii Nauk SSSR Ser. Mat. vol. 11 (1947) pp. 411-504 (Russian).

6. R. Godement, Théorie générale des sommes continues d'espaces de Banach, C. R. Acad. Sci. Paris vol. 228 (1949) pp. 1321-1323.

7. N. Jacobson, The radical and semi-simplicity for arbitrary rings, Amer. J. Math. vol. 67 (1945) pp. 300-320.

8. - A topology for the set of primitive ideals in an arbitrary ring, Proc. Nat. Acad. Sci. U.S.A. vol. 31 (1945) pp. 333-338. 
9. - Structure theory for algebraic algebras of bounded degree, Ann. of Math. vol. 46 (1945) pp. 695-707.

10. I. Kaplansky, Dual rings, Ann. of Math. vol. 49 (1948) pp. 689-701.

11. - Groups with representations of bounded degree, Canadian Journal of Mathematics vol. 1 (1949) pp. 105-112.

12. — Normed algebras, Duke Math. J. vol. 16 (1949) pp. 399-418.

13. - Topological representation of algebras, II, Trans. Amer. Math. Soc. vol. 68 (1950) pp. 62-75.

14. F. Murray and J. von Neumann, On rings of operators, Ann. of Math. vol. 37 (1936) pp. 116-229.

15. M. Neumark, Rings with involutions, Uspehi Matematičeskih Nauk (N. S.) vol. 3 (1948) pp. 52-145.

16. J. von Neumann, On rings of operators. Reduction theory, Ann. of Math. vol. 50 (1949) pp. 401-485.

17. D. Raikov, To the theory of normed rings with involutions, C. R. (Doklady) Acad. Sci. URSS. vol. 54 (1946) pp. 38i-390.

18. C. Rickart, Banach algebras with an adjoint operation, Ann. of Math. vol. 47 (1946) pp. 528-550.

19. I. Segal, Irreducible representations of operator algebras, Bull. Amer. Math. Soc. vol. 53 (1947) pp. 73-88.

20. Hing Tong, On ideals of certain topologized rings of continuous mappings associated with topological spaces, Ann. of Math. vol. 50 (1949) pp. 329-340.

Institute For Advanced Study,

Princeton, N. J.

University of Chicago, Chicago, Ill. 